\title{
Stochastic Non-Pharmaceutical Optimal Control Strategies to Mitigate COVID-19
}

This paper was downloaded from TechRxiv (https://www.techrxiv.org).

LICENSE

CC BY-NC-SA 4.0

SUBMISSION DATE / POSTED DATE

$14-04-2021 / 19-04-2021$

\section{CITATION}

Scarabaggio, Paolo; Carli, Raffaele; Cavone, Graziana; Epicoco, Nicola; Dotoli, Mariagrazia (2021): Stochastic Non-Pharmaceutical Optimal Control Strategies to Mitigate COVID-19. TechRxiv. Preprint. https://doi.org/10.36227/techrxiv.14413259.v1

$\mathrm{DOI}$

10.36227/techrxiv.14413259.v1 


\title{
Stochastic Non-Pharmaceutical Optimal Control Strategies to Mitigate COVID-19
}

\author{
Paolo Scarabaggio, Graduate Student Member, IEEE, Raffaele Carli, Member, IEEE, \\ Graziana Cavone, Member, IEEE, Nicola Epicoco, and Mariagrazia Dotoli, Senior Member, IEEE
}

\begin{abstract}
This paper proposes a stochastic non-linear model predictive controller to support policy-makers in determining robust optimal strategies to tackle the COVID-19 secondary waves. First, a time-varying SIRCQTHE epidemiological model (considering Susceptible, Infected, Removed, Contagious, Quarantined, Threatened, Healed, and Extinct compartments of individuals) is defined to get reliable predictions on the pandemic dynamics on a regional basis. A stochastic Model Predictive Control problem is then formulated to select the necessary control actions to minimize the arising socio-economic costs. In particular, considering the unavoidable uncertainty characterizing this decisionmaking process, we ensure that the capacity of the network of regional healthcare systems is not violated in accordance with a chance constraint approach. Furthermore, since the infection rate depends on people's mobility, differently from the related literature, we model the control actions as interventions affecting the mobility levels associated to different socio-economic categories. The effectiveness of the presented method in properly supporting the definition of diversified regional strategies for tackling the COVID-19 spread is tested on the network of Italian regions using real data from the Italian Civil Protection Department. However, provided the availability of reliable data, the proposed approach can be easily extended to cope with other countries' characteristics and different levels of the spatial scale.
\end{abstract}

Note to Practitioners-This paper is motivated by the emerging need for developing effective methods to support policy-makers in efficiently mitigating the effects of COVID-19 pandemic contagions. The proposed feedback control strategy - combining a multi-region epidemiological SIR-based model with a nonlinear stochastic Model Predictive Control (MPC) - allows the decision-makers to robustly identify the most effective restrictive measures taking into account the corresponding effects on the healthcare and socio-economic systems. The proposed framework is a general and flexible method that can be applied to various real scenarios, leveraging the mobility data available in the Google mobility service to recognize patterns and predict future behaviors of individuals. The presented strategy can be implemented in any decision support system or engineering software for emergency management, providing decision-makers with an effective Information and Communication Technology tool for the optimal planning of the necessary control actions to minimize socio-economic costs considering the unavoidable

Manuscript received on. (Corresponding author: P. Scarabaggio.)

The work of M. Dotoli is partially supported by Italian University and Research Ministry under project RAFAEL (National Research Program, contract n. ARS01-00305).

P. Scarabaggio, R. Carli, G. Cavone, and M. Dotoli are with the Department of Electrical and Information Engineering of the Polytechnic of Bari, Italy. (e-mail: \{paolo.scarabaggio, raffaele.carli, graziana.cavone, mariagrazia.dotoli\}@poliba.it).

$\mathrm{N}$. Epicoco is with the Department of Information Engineering, Computer Science and Mathematics (DISIM) and with the Center of Excellence DEWS (Design methodologies for Embedded controllers, Wireless interconnect and System-on-chip), University of L'Aquila, L'Aquila, Italy (e-mail: nicola.epicoco@univaq.it). uncertainty in the pandemic model parameters. By changing the characteristic tuning parameters of the controller decisionmakers can enforce different behaviors while ensuring that the capacity of the healthcare system is not violated.

Index Terms-COVID-19, pandemic modeling, epidemic control, mitigation strategies, stochastic Model Predictive Control.

\section{Introduction AND PAPER Positioning}

0 INCE the end of 2019, in about a year the coronavirus SARS-CoV-2 (commonly called as COVID-19) has exceeded 1.7 million deaths worldwide, with more than 80 million of confirmed cases in 222 countries, areas, or territories, thus resulting in one of the most impacting pandemics in the recent decades [1]. As currently the vaccinations against this virus have not yet completed the mass coverage, the main control action still relies in the adoption of some NonPharmaceutical Interventions (NPIs), which basically consist in mobility restrictions, social-distancing, and use of masks and face coverings by citizens [2].

The recent research trends in the COVID-19 framework have been devoted to disease transmission modeling and control, with the aim of suppressing, or at least mitigating, the spread of infections [3], [4]. In particular, while several studies focus on the epidemiological modeling, less attention has been devoted to the development of control strategies [4], [5]. The next subsections detail the research trends of the two research topics.

\section{A. Dynamical Modeling of COVID-19 Pandemic}

The description of the pandemic dynamics can take advantage of the existing compartmental epidemiological models [5]. Hence, not surprisingly, several models are nowadays available in the literature to describe the COVID-19 dynamics. Basically, all the presented models are variants of the classical $S I R$-based epidemiological model, which considers the compartments of Susceptible, Infective, and Recovered individuals: further compartments are in turn added to suitably dividing the population into classes so that the corresponding flow patterns among them can be considered. An overview on SIR-based epidemiological models in the field of COVID-19 is presented in [6].

In particular, since one of the main characteristics of COVID-19 is that many of the persons who contract the disease are asymptomatic, a SIDARTHE model dividing the population into Susceptible, Infected, Diagnosed, Ailing and 
Recognized, Threatened, Healed, and Extinct is presented in [3] for predicting transmission dynamics of the COVID-19 pandemic in Italy. Similarly, the contribution in [7] presents a SEIAHR model for Ethiopia.

Another group of works deepens the analysis at a regional level, thus allowing to take into account the economic and social differences existing within almost any country [4], [8]. Among the literature contributions addressing the spatial dynamics of the COVID-19 epidemic, a SIR-based model is presented in [9] to describe the virus dynamics at the country level and a subsequent local model referred to a single node of the health system network to model the flows of patients at the level of a regional hospital care center for COVID-19 infected patients. The contribution in [10] divides the society into a set of relatively homogeneous regions proposing a $S A I R$ model for each region. In addition, a SEIR model is presented in [11] to support planning the Italian inter-regional mobility, while a SEIIR model is proposed in [12] to assess how the school closure and telework have impacted some French regions during the COVID-19 lockdown. Furthermore, a multiregion $S I R$ model and its $S I_{2} R_{2}$ extension is suggested in [13]. Finally, the Italian regional healthcare systems' characteristics are considered in [8], where a model dividing the population into six compartments is presented.

\section{B. Control Strategies for COVID-19 Mitigation}

The above recalled contributions still present a relevant limitation. In fact, although some of the recalled mathematical models are used to investigate the effects of different NPIs strategies (mainly through scenario-based simulations), they typically do not provide a feedback control method to properly identify the most effective action(s) in a dynamical framework [14]. In a nutshell, these approaches aim to contain the pandemic not take into account the current state of the system [5], which conversely is crucial since severe lockdown measures clearly cause relevant socio-economic damages.

As a matter of fact, actually, only a few literature contributions exist on the definition of proper control actions to mitigate the COVID-19 spread. Among these, the work in [15] proposes a classical SIR model to test on/off social distancing measures aimed at minimizing the number of symptomatic infectious individuals and the duration of the social distancing policies, while considering the occupancy of Intensive Care Units (ICUs). Similarly, the work presented in [16] proposes a fast switching policy, where multi-shot interventions are adopted to switch between social isolation and work days depending on the outcomes of two different predictive models.

A more advanced solution to tackle the COVID-19 control problem relies on the use of Model Predictive Control (MPC), which includes both feedback control and optimization, thus allowing to take into account the deviations of the predictive model from the real evolution of the pandemics [17], and hence the unavoidable uncertainty in the model provided that the values of the control parameters are carefully chosen [18]. In fact, since defining the proper strategies to mitigate the COVID-19 spread is a strategic decision making process, the large amount of computational resources typically required by the MPC controller is not an issue [19], [20].
Nevertheless, only a few papers have investigated the use of MPC in the COVID-19 framework. Robust MPC in combination with a SIDARTHE epidemiological model is proposed in [14] to determine proper social distancing measures in Germany so that the number of fatalities caused by COVID19 over time and the economic cost of the measures are minimized. An optimal dynamic control strategy aimed at minimizing the social and economic cost of controlling the pandemic in the US while ensuring that the maximum number of infected people remains under a given peak value is proposed in [21] together with a SEIAR model, in which the values of the unmeasured or hidden states are estimated through the discrete unscented Kalman filter. Moreover, an MPC technique aimed at reaching herd immunity with the minimum age-dependent social distancing measure in Germany and while considering the ICU occupancy is proposed in [22] in conjunction with a SEITPHR model. Finally, the previous contribution by the authors in [4] proposes the joint use of a SIRQTHE model (that is, considering the compartments of Susceptible, Infected, Removed, Quarantined, Threatened, Healed, and Extinct) and of non-linear MPC to minimize the cost of the mitigation strategies on a regional basis, while ensuring that the capacity of the network of regional healthcare systems is not violated.

Throughout most of the recalled models and control solutions, a perfect knowledge of parameters (e.g., on recovery rates, state information, and network structures) is assumed. Nevertheless, acknowledging uncertainty is an important issue in the context of epidemics [5].

\section{Paper Contribution}

Within the above described background, this paper revises and extends the recalled contribution in [4] by presenting a time-varying SIR-based epidemiological model in conjunction with a non-linear stochastic MPC approach. The proposed approach is able to define the specific strategies undertaken at the regional level take into account the uncertainty of the model parameters. In particular, we adopt a chanceconstrained optimization technique to consider the variation of the spreading parameters through the optimization process. More in detail, we refer to control actions able to mitigate the effect of secondary waves of the pandemic assuming the inefficiency of primary NPIs (i.e., for instance, socialdistancing measures and use of face masks in public). Using the real data from the Italian Civil Protection Department [23], we analyze the Italian scenario, where the healthcare system (called Servizio Sanitario Nazionale) is regionally based and enormously varies from region to region [24].

Compared with other existing literature contributions in the field of COVID-19, the novelties of this work are as follows:

1) We define a SIRCQTHE model to describe the spread of COVID-19. Differently from most contributions like [9][11] where the authors propose simple compartmental models, we propose a more accurate epidemic model since we consider eight compartments (namely, Susceptible, Infected, Removed, Contagious, Quarantined, Threatened, Healed, and Extinct) that fully describe the epidemic dynamics. Nevertheless, differently from more detailed models presented in the 
related literature, we introduce several simplifying assumptions that allow a realistic application of the proposed model. In particular, differently from [3] we disregard the difference between asymptomatics and symptomatics individuals as in most countries data related to these two categories is absent or unreliable while differently from the model in [8], we split the compartment of the recovered individuals into two classes, that is, Healed (i.e., recognized individuals that heal after a transition in the status of Quarantined), and Removed (i.e., never detected people). Furthermore, we improve the previous contribution [4] by proposing a novel epidemiological model that better fits the COVID-19 secondary waves in the current scenario. In fact, the model in [4] suitably represents the dynamics of an epidemic in the early stage. In contrast, the novel model shows its potentialities with the epidemic's progress when more accurate data become available. In particular, we divide the Infected individuals into Contagious and Infected yet not contagious, and we assume that Threatened people must observe a quarantine period when recovered. Moreover, we disregard the time dependency of the death rate, and we model its relation with the number of Threatened people. It is essential to remark that, even employing several classes, in our model, we assume only a pure constant delay between the Infected and the Contagious individuals. At the same time, three parameters (of the nine in totals) belong to terminal compartments, thus easily identifiable with raw data. In addition, while other contributions dynamically identify the model parameters over a shifting subset of the fitting period, in our work time-varying functions are presented to model the timevarying parameters. To the best of the authors' knowledge, the proposed SIRCQTHE model is the only compartmental model that leverages the Google Mobility Reports in [25] to better describe the time dependency of the infection rate. At the same time, we use the ratio between new daily discovered cases and the daily swabs to consider the variability of the rate of Contagious people identified and Quarantined.

2) As regards the control approach and differently from the aforementioned literature [14]-[16], where the proposed control actions aim at minimizing the number of fatalities or at ensuring that the healthcare system is not overloaded, our work also takes into account the impact of the control strategies on the economic framework. In fact, the proposed approach aims at reducing the cost of mitigation strategies while respecting the maximum capacity of the healthcare system. Moreover, differently from other existing related works, we model the control actions as interventions affecting the mobility levels associated with different socio-economic categories, since the infection rate heavily depends on people's mobility [26], [27].

3) Since the proposed model is highly non-linear, the accurate identification of characteristic parameters is challenging. Hence, uncertainty in the model parameters should be analyzed. For instance, the authors in [14] develop a robust MPC-based feedback strategy using interval arithmetic that takes uncertainty into account. In particular, the work proposes a framework where the state estimation is biased with known bounds. However, due to the difficulty of deriving reliable bounds on all parameters in the model, it only focuses on the infection rate with an uncertainty of $\pm 5 \%$. Conversely, our work considers variability and uncertainty in all the model parameters. We propose a stochastic optimal control problem that intrinsically considers uncertainty on each parameter based on specific bounds. Moreover, it focuses on control of the number of fatalities while we enforce the number of Threatened people under a safety threshold by defining a chance-constrained formulation for the optimization problem that ensures that the probability of meeting the specific constraint is above a certain acceptable level of risk.

4) The application of our approach to the Italian scenario shows its effectiveness and flexibility in supporting the definition of diversified regional strategies for managing the COVID-19 disease under different governmental policies. In this context, our contribution is twofold. On the one hand, thanks to the proposed dynamical identification procedure, we show the advantages of our model in describing and predicting the COVID-19 secondary waves using real data from the Italian Civil Protection Department. On the other hand, we discuss the results achieved by the proposed stochastic MPC scheme by performing several long-term simulations.

\section{Paper Organization}

The rest of this work is structured as follows. Section II presents the SIRCQTHE model and its dynamical equations. Section III proposes the stochastic MPC framework, describing the corresponding control variables, objectives, and constraints, and formulating the comprehensive stochastic optimal control problem. Section IV reports the numerical outcomes achieved by the simulations based on the real data of the Italian regions. Finally, Section V concludes the paper highlighting possible future developments. Appendix A details the parameters identification procedures for the SIRCQTHE model.

\section{Model of The COVID-19 Dynamics}

Typically, compartmental models are considered appropriate to model the proliferation of a virus within a large population. In these models, the population is partitioned into compartments and can flow from one compartment to another according to specific rates.

Several models can be found in the pertinent literature to adapt the classical SIR model to the specific features of the various pandemics, typically by considering additional compartments and by better modeling their interactions [6].

In this paper, a novel time-varying discrete-time epidemiological model for the COVID-19 spread is proposed, named SIRCQTHE, which classifies individuals into eight classes.

More in detail, the overall population is divided into eight compartments that correspond to the state variables of the model. Denoting by a Latin letter each state variable, and denoting the time step as $k$, the population is divided into:

- Susceptible individuals $\tilde{S}(k)$ : this class represents people that can be infected;

- Infected individuals $\tilde{I}(k)$ : this class represents infected (undetected) but not yet contagious people; 
- Removed individuals $\tilde{R}(k)$ : this class represents infected (undetected) people that are recovered and thus not contagious;

- Contagious individuals $\tilde{S}(k)$ : this class represents contagious (undetected) people that can infect other individuals;

- Quarantined individuals $Q(k)$ : this class represents infected (detected) people that are currently isolated;

- Threatened individuals $T(k)$ : this class represents hospitalized people in a life-threatening or noncritical situation;

- Healed individuals $H(k)$ : this class represents infected (detected) people that are recovered and thus not contagious;

- Extinct individuals $E(k)$ : this class represents dead individuals.

The state variables indicated with a tilde cannot be directly observed with reasonable confidence, since no official data is available.

We remark that the proposed model aims at coping with the urgent need of representing and predicting the COVID-19 pandemic diffusion with a high level of accuracy. As shown in Section I, various papers aimed at representing the dynamics of the COVID-19 pandemic present different compartmental models, but they generally lack an accurate identification of the model parameters since this is a challenging task.

Therefore, in order to perform a fruitful parameters identification and get reliable forecasting, we employ a highly data-driven approach defining a model that takes the available epidemiological data into account. The proposed model allows representing almost all the facets of the pandemic diffusion since it comprehends more compartments than most of the other compartmental models proposed for COVID-19 modeling. However, thanks to several data-based simplifying assumptions, we preserve the model's applicability to any scenario.

In particular, as for other compartmental models, we assume that the pandemic dynamics is faster than the dynamics of birth and death; therefore, such events can be considered negligible. In addition, based on the current level of knowledge, we reasonably assume that the probability of becoming susceptible after being healed is negligible too, i.e., this dynamics is much lower than the main time constant of the pandemic [28]. In the SIRCQTHE model we compress or eliminate some of the classes that are often considered, and we disregard some connections between the compartments. For instance, we consider a single class for Quarantined people, without distinguishing between asymptomatics and symptomatics. In fact, in most countries, data on Quarantined people's health conditions are absent or unreliable. Following the current regulations, we assume that Threatened people must observe a quarantine period when recovered. Moreover, although several models include a class for people requiring intensive care treatments and a class for people requiring general clinical treatments, we consider a single compartment for hospitalized people. In fact, statistical data show that the above compartments are correlated with a 0.1 ratio, i.e., people requiring general treatments to combat the COVID-19 symptoms are ten times those requiring intensive care treatments. Moreover, we divide the recovered individuals into the Removed and the Healed compartments, since official data are available only for the latter class. Lastly, we employ time-varying functions to model time-varying parameters. In fact, as the pandemic is continuously evolving, we cannot rely on constant parameters.

Despite these simplifying assumptions, as shown in Appendix A, the presented SIRCQTHE model is effective in the identification phase by only requiring a minimal set of epidemiological data, which are typically available in most occidental countries.

The SIRCQTHE model is thus composed of eight timevarying difference equations, which represent the dynamics of individuals' flows between the various compartments. We consider as state variables of the model the fraction of the overall population related to the various compartments. The model can be written as follows:

$$
\begin{aligned}
\tilde{S}(k+1) & =\tilde{S}(k)-\beta(k) \tilde{C}(k) \tilde{S}(k) / N \\
\tilde{I}(k+1) & =\tilde{I}(k)+\beta(k) \tilde{C}(k) \tilde{S}(k) / N-\rho \tilde{I}(k) \\
\tilde{R}(k+1) & =\tilde{R}(k)+\gamma \tilde{C}(k) \\
\tilde{C}(k+1) & =\tilde{C}(k)+\rho \tilde{I}(k)-(\gamma+\theta(k)+\lambda) \tilde{C}(k) \\
Q(k+1) & =Q(k)+\theta(k) \tilde{C}(k)+\pi T(k)-(\delta+\mu) Q(k) \\
T(k+1) & =T(k)+\mu Q(k)+\lambda \tilde{C}(k)-(\pi+\varepsilon(T(k))) T(k) \\
H(k+1) & =H(k)+\delta Q(k) \\
E(k+1) & =E(k)+\varepsilon(T(k)) T(k)
\end{aligned}
$$

where $N$ represents the whole population.

The overall interconnections between the above compartments are shown in Fig.1. The eight classes are connected by directed arcs whose weights correspond to the parameters that put in relation the corresponding classes. In particular, $\beta(k) \in \mathbb{R}_{+}$is the time-varying infection rate, whose values depend on the population behavior and on the adopted social distancing measures. Moreover, $\theta(k) \in \mathbb{R}_{+}$is the timevarying detection rate that describes the rate of Contagious people that are recognized and Quarantined; this must be modeled as a time-varying parameter since it largely depends on the epidemiological situation. As will be demonstrated in Appendix A, the parameter $\beta(k)$ can be correlated with people's mobility through the use of the Google Mobility Reports [25] and the parameter $\theta(k)$ with the ratio between new daily discovered cases and the daily swabs. The parameter $\rho \in \mathbb{R}_{+}$is the so-called incubation rate. Parameters $\gamma \in \mathbb{R}_{+}$ and $\delta \in \mathbb{R}_{+}$are the so-called healing rates. However, the first describes the rate of healing of Contagious and unrecognized people (thus, not requiring hospitalization) that are no more infectious. In contrast, the second is the healing rate of Quarantined people who can leave this class when they are no more infectious or legally obligated to stay at home. Parameters $\lambda \in \mathbb{R}_{+}$and $\mu \in \mathbb{R}_{+}$are the hospitalization rates: $\lambda$ is the rate of people recognized and immediately hospitalized when a severe symptomatic condition occurs, while $\mu$ is the rate of Quarantined people that need for hospitalization. The parameter $\pi \in \mathbb{R}_{+}$is the healing rate of Threatened people, here we assume that after released from the hospital, people must observe a quarantine period. Lastly, $\varepsilon(T(k)) \in \mathbb{R}_{+}$is 


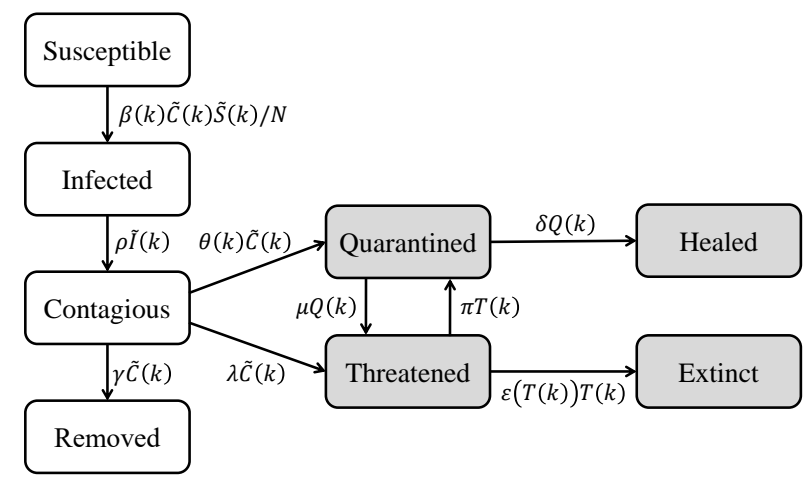

Fig. 1. Scheme of the SIRCQTHE model: the compartments whose state is directly observable are indicated by filled rectangles.

the time-varying death rate. The latter is defined as a timevarying parameter dependent on the number of Threatened people. The assumption made on each parameter and their relation is discussed in Appendix A.

\section{Chance Constrained Predictive Control of THE COVID-19 OUTBREAK}

Before introducing the proposed stochastic MPC framework, we first remark that this work aims at supporting policymakers in identifying robust optimal mitigation strategies to tackle future pandemic secondary waves. In particular, we assume that the basic NPIs actions (e.g., the use of face masks and the measures aimed at social-distancing) are ineffective or their application have been relaxed. Consequently, as vaccination requires a long time to achieve the mass coverage, any long term control of the COVID-19 spread is assumed to be focused on reaching heard immunity; however, any short term mitigation strategy simultaneously should ensure that the healthcare capacity is not violated and the economic loss is minimized. This goal can be reached by effectively applying and optimally controlling some more restrictive interventions than the basic NPIs [2]. Consequently, in this section, we present a stochastic optimal control framework which supports decision-makers in determining the most effective strategies to be undertaken during secondary wave mitigation phases.

As for the receding horizon scheme, in the remainder of the paper we assume that the prediction and control horizon have the same length [29]. Specifically, at the generic sampling step $h \in \mathbb{Z}_{+}$the horizon - defined as $\mathcal{K}(h)=\{h, \ldots, k, \ldots, h+$ $K-1\}$ - contains $K$ equally spaced time slots with length $\Delta_{k}$.

\section{A. Model of control actions}

In this section we introduce the different control actions that could adopted to tackle the consequences of the COVID19 outbreak. First, we assume that no structural parameter related to the healthcare system can be directly affected by the control action. Conversely, we assume that any control action is focused only on reducing the parameter $\beta(k)$, i.e., the infection rate. Indeed, this parameter is somehow controllable. For example, a region lockdown would significantly reduce the corresponding infection rate. In this context, following [26], [27] and [30], we assume that the infection rate is strongly influenced by people's mobility in different socioeconomic categories. Hence, let us denote by $\mathcal{G}=\{1, \ldots, G\}$ the set of socio-economic categories, e.g., transport, retail, restaurants, workplaces. We assume that the infection rate can be decomposed into different terms each of which is mainly affected by a given category. Given the results obtained in several preliminary experiments on real data, we consider that a reduction of the mobility associated with the different socio-economic categories can be, with a good approximation, represented with a linear decrease of the infection rate as follows:

$$
\beta(k)=\beta_{0}+\boldsymbol{\beta}^{\top} \mathbf{m}(k), \quad \forall k \in \mathcal{K}(h)
$$

where $\beta_{0}$ is the infection rate not explicable with a variation of the mobility (e.g., household infection), $\boldsymbol{\beta}=\left(\beta_{1}, \ldots, \beta_{G}\right)^{\top}$ is the column vector collecting the term $\beta_{g}$ of the infection rate corresponding to each socio-economic category $g \in \mathcal{G}$, and $\mathbf{m}(k)=\left(m_{1}(k), \ldots, m_{G}(k)\right)^{\top}$ is the column vector collecting the mobility level $m_{g}(k)$ at time step $k$ for each socioeconomic category $g \in \mathcal{G}$. We assume that $m_{g}(k)=1$ when the $g$-th category's mobility level equals the nominal value, i.e., the value in absence of mobility restrictions.

Let us preliminarily define a vector of control variables $\mathbf{u}:=$ $\left(\mathbf{u}(h)^{\top}, \ldots, \mathbf{u}(h+K-1)^{\top}\right)^{\top}$ that models the interventions that affect the mobility rate associated to all the $G$ socio-economic categories over the given control horizon: the control action at time step $k$ is denoted as $\mathbf{u}(k):=\left(u_{1}(k), \ldots, u_{G}(k)\right)^{\top}$. Hence, we correlate the infection rate coefficients with the activity restriction measures for each time step $k$ in accordance with the following linear equation:

$$
\beta(k)=\beta_{0}+\boldsymbol{\beta}^{\top}(1-\mathbf{u}(k)), \quad \forall k \in \mathcal{K}(h) .
$$

We assume that the control action $u_{g}(k)$ related to the restriction of activities in the $g$-th socio-economic category at time step $k$ gets a value in the interval $[0,1]$. We also assume that $u_{g}(k)$ cannot be chosen independently from the other control actions $u_{i}(k), \forall i \neq g$. For instance, a restrictive measure that aims at reducing the mobility in the retail category will also affect mobility in the transport category. Therefore, since any restrictive measure applied by the government has at the same time impacts on different socio-economic categories, we assume that the control action $\mathbf{u}(k)$ gets values in the discrete set $\mathcal{U}:=\left\{\boldsymbol{v}_{1}, \ldots, \boldsymbol{\nu}_{P}\right\}$ with cardinality $P$. The control system can impose the proposed policies by properly defining the constraint set on the given decision variable:

$$
\mathbf{u}(k) \in \mathcal{U}:=\left\{\boldsymbol{\nu}_{1}, \ldots, \boldsymbol{\nu}_{P}\right\}, \quad \forall k \in \mathcal{K}(h) .
$$

It should be noted that, in order to avoid too frequent and impractical changes in the strategies, the control actions can be kept constant over a given period equal to $\Delta_{l}=\omega \Delta_{k}$ (i.e., for $\omega$ time slots). For instance, if $\Delta_{k}$ corresponds to one day, it could be meaningful to set the periodicity of the control actions equal to one week (i.e., $\omega=7$ ). Assuming that $K=L \omega$, with $L \in \mathbb{N}$, the following additional constraints on the control actions are then introduced to keep the control actions constant in each period $l$ :

$\mathbf{u}(\omega l+1)=\mathbf{u}(\omega l+2)=\cdots=\mathbf{u}(\omega l+\omega), \forall l=0, \ldots, L-1$. 


\section{B. The Proposed Stochastic Optimal Control Problem}

The proposed MPC approach aims at optimizing the cost of the mobility reduction, i.e., the impact of the restrictive measures on the economic framework, while respecting a threshold on the number of ailing people. Let us preliminarily define $\phi=\left(\phi_{1}, \ldots, \phi_{G}\right)^{\top}$ as the column vector containing all the cost coefficients $\phi_{g}$ that represent the impact on the economic framework of the different categories. Thus, we define $\phi_{K}=\mathbf{1}_{K} \otimes \phi$, where $\otimes$ is the so-called Kronecker product.

We remark that we define the control actions computed at time $h$ over the whole control horizon (i.e., from time step $h$ to time step $h+K-1)$ as $\mathbf{u}:=\left(\mathbf{u}(h)^{\top}, \ldots, \mathbf{u}(h+K-1)^{\top}\right)^{\top}$, while we indicate the control actions computed at time $h-1$ over the whole control horizon (i.e., from time step $h-1$ to time step $h+K-2)$ with $\overline{\mathbf{u}}:=\left(\overline{\mathbf{u}}(h-1)^{\top}, \ldots, \overline{\mathbf{u}}(h+K-\right.$ $\left.2)^{\top}\right)^{\top}$.

In order to smooth the control action variation over time we define two additional vectors. In particular, we define a vector $\Delta \mathbf{u}$ which contains the differences between the control actions related to consecutive time steps of the control horizon as computed at the current time (i.e., $h)$ as $\Delta \mathbf{u}:=\left(\mathbf{0}_{G}^{\top},(\mathbf{u}(h+\right.$ $\left.1)-\mathbf{u}(h))^{\top}, \ldots,(\mathbf{u}(h+K-1)-\mathbf{u}(h+K-2))^{\top}\right)^{\top}$. Note that, in vector $\Delta \mathbf{u}$, we include $\mathbf{0}_{G}^{\top}$ as first block to make the overall vector size equal to the dimension of the control vector $\mathbf{u}$. Moreover, we define a vector $\Delta \overline{\mathbf{u}}$ containing the difference values between the control actions related to corresponding time steps of the control horizon as computed at the current time step (i.e., $h$ ) and at the previous one (i.e., $h-1$ ) as $\Delta \overline{\mathbf{u}}:=$ $\left(\mathbf{0}_{G}^{\top},(\mathbf{u}(h)-\overline{\mathbf{u}}(h))^{\top}, \ldots,(\mathbf{u}(h+K-2)-\overline{\mathbf{u}}(h+K-2))^{\top}\right)^{\top}$. Note that, in vector $\Delta \overline{\mathbf{u}}$, we do not include the terms related with time step $(h+K-1)$, since at each shift of the control horizon we apply the first action.

These vectors are used in the proposed cost function. In fact, the objective function denoted as $J(\overline{\mathbf{u}}, \mathbf{u})$ is composed by multiple cost terms as follows:

$$
J(\overline{\mathbf{u}}, \mathbf{u})=\phi_{K}^{\top} \mathbf{u}+\alpha_{1} \phi_{K}^{\top} \Delta \mathbf{u}+\alpha_{2} \phi_{K}^{\top} \Delta \overline{\mathbf{u}} .
$$

The cost function in (13) is composed of three parts. The first part is the term that takes the impact of restrictive measures into account. The second part is a regularization term: by tuning the coefficient $\alpha_{1}$, we smooth the variability of restrictive measures over the control horizon by acting on vector $\Delta \mathbf{u}$. In fact, we aim at reducing switches avoiding a bang-bang control action (i.e., avoiding that the solution jumps back and forth between the extreme values). For instance, by increasing $\alpha_{1}$, we avoid the solution jumping from $\mathbf{u}(h)=(1, \ldots, 1)^{\top}$ (i.e., full lockdown) to $\mathbf{u}(h+1)=(0, \ldots, 0)^{\top}$ (i.e., no lockdown). The third part of (13) is a memory term. In fact, by modifying the term $\alpha_{2}$, we aim at decreasing the time in which decisions taken in a previous time step are modified by the central authority in the subsequent time step by leveraging on vector $\Delta \overline{\mathbf{u}}$. We underline that the proposed approach aims at identifying the set of restrictive measures to be applied in a region over a time period. The vector of control actions $\overline{\mathbf{u}}$ comprehends the decision taken by an authority (e.g, a government) in the past and communicated to the population.
Therefore, a continuous switch of these decisions would be unrealistic in a practical application.

On the basis of the above defined control variables and related constraint set, the corresponding state model, and the objective function of the online optimization, the optimal control problem is defined as follows:

$$
\begin{array}{ll}
\underset{\mathbf{u}}{\operatorname{minimize}} & J(\overline{\mathbf{u}}, \mathbf{u}) \\
\text { subject to } & \text { SIRCQTHE model (1)-(8), } \forall k \in \mathcal{K}(h) \\
& \text { constraints (11)-(12), } \forall k \in \mathcal{K}(h) \\
& \mathbf{T}-T^{\max } \mathbf{1}_{K} \leq \mathbf{0}_{K}
\end{array}
$$

where $\mathbf{T}-T^{\max } \mathbf{1}_{K} \leq \mathbf{0}_{K}$ is the non-linear constraint that ensure the containment of Threatened cases under a safety threshold.

The optimization problem (14) has $P K$ integer decision variables; furthermore, it presents non-linearities in the state model. Consequently, it is a mixed-integer non-linear programming (MINLP) problem.

However, by defining the problem in the above way, we cannot take into account the variability and uncertainty in the model parameters. In fact, as the model is non-linear, the identification of the parameters is nontrivial. Moreover, the data related to the different categories are scarce or nonhomogeneous or unavailable.

In order to include uncertainty in the problem, several approaches are available. However, since the aim of this contribution is to keep the number of Threatened people below a safety threshold by including a constraint for the optimization problem, we employ the so-called chance-constrained approach, which is one of the most commonly adopted techniques to solve optimization problems under uncertainties [31]. In detail, the chance-constrained formulation of an optimization problem ensures that the probability of meeting a specific constraint is above a certain level. Hence, we do not require to satisfy a specific constraint, but we settle it in a probabilistic way. By regrouping the SIRCQTHE model's parameters in $\boldsymbol{\xi}=\left(\beta_{0}, \boldsymbol{\beta}, \rho, \gamma, \theta, \lambda, \delta, \mu, \pi, \varepsilon\right)$, we can rewrite the aforementioned optimal control problem as:

$$
\begin{array}{ll}
\underset{\mathbf{u}}{\operatorname{minimize}} & J(\overline{\mathbf{u}}, \mathbf{u}) \\
\text { subject to } & \text { SIRCQTHE model (1)-(8), } \forall k \in \mathcal{K}(h) \\
& \text { constraints (11)-(12), } \forall k \in \mathcal{K}(h) \\
& \mathbb{P}\left\{\mathbf{T}(\boldsymbol{\xi})-T^{\max } \mathbf{1}_{K} \leq \mathbf{0}_{K}\right\} \geq 1-\epsilon
\end{array}
$$

where $\mathbb{P}\left\{\mathbf{T}(\boldsymbol{\xi})-T^{\max } \mathbf{1}_{K} \leq \mathbf{0}_{K}\right\}$ is the probability of satisfying the constraint. Moreover, we denote with $\epsilon \in[0,1]$ the risk level that the decision-maker is willing to accept. Note that we underline the dependence from $\xi$ of the Threatened cases.

Probabilistic optimization problems are hard to solve except in a few special cases. Most of the time is because the quantity $\mathbb{P}\left\{\mathbf{T}(\boldsymbol{\xi})-T^{\max } \mathbf{1}_{K} \leq \mathbf{0}_{K}\right\}$ is hard to compute and because the feasibility solution set defined by probabilistic constraints is generally nonconvex. However, we can approximately solve this problem using the Sample Approximation Approach (SAA) based on Monte Carlo samplings of the 
model's parameters $\boldsymbol{\xi}$. In fact, we can substitute the constraint with its approximated counterpart by:

$$
\frac{1}{M} \sum_{m=1}^{M} \mathbb{I}\left(\mathbf{T}\left(\boldsymbol{\xi}_{m}\right)-T^{\max } \mathbf{1}_{K} \leq \mathbf{0}_{K}\right) \geq 1-\epsilon
$$

where $\mathbb{I}(\cdot)$ denotes the indicator function which takes value 1 when the constraint is fulfilled and 0 otherwise. Moreover, $\boldsymbol{\xi}_{m}$ is a sampling of the model parameters based on their probability density function (PDF), and $M$ is the number of samplings. The SAA technique is mainly based on having a prior known PDF, from which $M$ independent and identically distributed (IID) random samples of the stochastic variables are obtained. In our case, we obtain the $M$ samples using a specific PDF for each model parameter.

\section{The MPC approach}

We propose a stochastic predictive control approach that aims at minimizing the impact of the mitigation strategies on the economic framework taking into account a safety threshold on the number of threatened people.

The control scheme - based on combining the MPC approach with the prediction of the COVID-19 dynamics (i.e., the SIRCQTHE model) - is shown in Figure 2 and it is described in detail in the sequel.

Following the predictive control theory, MPC is based on iterative, finite-horizon optimization of the plant model, where the change in the controlled variables of the modeled system is induced by changes in the manipulated variables. According to the rolling horizon method, the optimization problem is solved at each $\omega$ time steps in an iterative manner, based on the most recent input data. In particular, the proposed approach aims at minimizing the economic cost of social restrictions. In addition, as a core component, the MPC optimizer employs the SIRCQTHE dynamical model to predict the pandemic evolution and thus to keep the number of hospitalized people under a safety threshold. The obtained control actions (i.e., the mobility restrictions) related to the first $\omega$ time steps are applied to the system to steer its behavior to the desired one, while the horizon is shifted forward. Then, a new optimization problem is solved over the shifted prediction horizon, based on the updated information on forecasts and system states.

It must be noticed that the presented framework is a closedloop feedback control technique that may depend on quantities that are both directly and not directly measurable. In effect, at each time step, an identification procedure should be performed in order to estimate the parameters of the SIRCQTHE model. In particular, the identification procedure consists of using, at each time step, the most recent epidemiological data (such as, for instance, the number of Hospitalized or Quarantined person) and the mobility trends to dynamically update the remaining SIRCQTHE parameters (see, e.g., in Appendix A the dynamical identification algorithm referred to the Italian scenario).

Predicting the characteristic variables that influence the epidemic dynamics can be challenging since several variables are not directly observed by sensors and the presence of disturbances influences the accuracy of the model response.

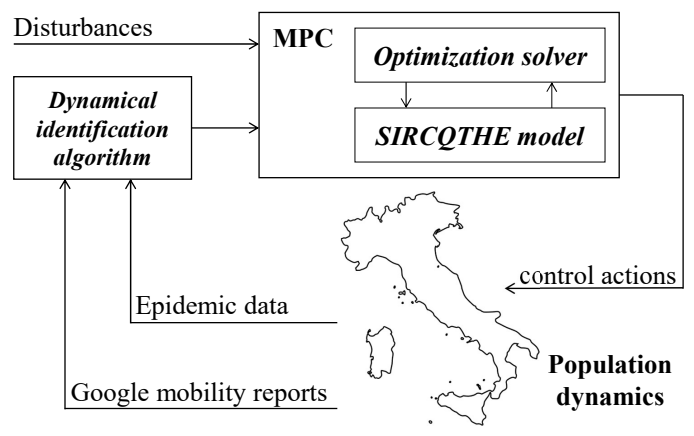

Fig. 2. Schematization of relations between the control action periodicity and the length of time horizon intervals.

In this context, the proposed stochastic MPC framework can limit such uncertainties thanks to a probabilistic constraint on the maximum number of Threatened people and to a rolling horizon approach where we compute the feedback control actions based on the periodic update of the state of the system and the consequent prediction of its evolution.

\section{NumericAl EXPERIMENTS BASED ON THE ITALIAN EPIDEMIOLOGICAL SCENARIO}

In order to assess the effectiveness of the proposed stochastic optimal control approach, in this section we apply the methodology on the Italian scenario of the COVID-19 outbreak. In particular, we report and analyze the optimal mitigation strategies determined by solving the stochastic optimal control problem (15) for each of the 20 Italian regions. In effect, the Italian scenario well fits the framework addressed by the proposed control approach since the national healthcare system is regionally based. Indeed, regions have healthcare facilities with different quality levels; moreover, they adopt diverse regulations and procedures concerning hospitalization, therapy, swabs, and prevention during the pandemic.

In the sequel, we first describe the estimation of the model parameters for the SIRCQTHE model based on the real data available for the Italian pandemic. Then, we discuss the outcomes that are provided by the proposed methodology.

\section{A. Set-up of Experiments}

In this section, we report the main outcomes of the dynamical identification procedure, together with the initial conditions for the considered control approach. For the detailed description of the fitting procedure, the reader is referred to Appendix A.

The proposed MPC approach is tested over a simulation period of 10 weeks starting from December 6, 2020, using a prediction horizon of six weeks. The sampling interval $\Delta_{k}$ is set to one day (i.e., $K=42$ ). A daily implementation of the restrictive measures would be unrealistic in a real-life scenario, therefore, we assume weekly based control actions (i.e., $L=6$ and $\Delta_{l}=\omega \Delta_{k}$, with $\omega=7$ ). We set the initial number for the classes Quarantined, Threatened, Recovered, and Extinct to the values of December 5, 2020, according to the real data available in [23].

We implement the stochastic optimal control problem (15) in MATLAB [32] employing the Global Optimization toolbox 
on a laptop with a $1.3 \mathrm{GHz}$ Intel Core i7 CPU and $8 \mathrm{~GB}$ RAM. Problem (15) is a MINLP problem; thus, its resolution is challenging mainly due to its combinatorial complexity and non-linearity. In order to solve such a problem, we employ a two-step genetic algorithm (GA) based on the MATLAB GA [33]. In the first step, denoting by $n$ the number of control variables, we perform $1,000 \mathrm{n}$ parallel computations of the MATLAB GA with 1,000 $n$ generations (i.e., an initial population size of $1,000 n$ ). In the second step, we use as the initial population for an additional run of the MATLAB GA optimization process the outcome of the first step.

The maximum number of Threatened people $T^{\max }$ is specifically defined for each region due to the high heterogeneity of the Italian healthcare system. In particular, for each region we set $T^{\mathrm{max}}=\alpha_{3} T^{\mathrm{tot}}$, where $T^{\mathrm{tot}}$ is the sum of the number of ICUs and Noncritical Care Beds (NCBs) reserved for COVID19 cases as defined by the Italian Ministry of Health's protocol [34]. Note that assuming a threshold lower than the maximum number of beds is reasonable and realistic since this is one of the indicator values set up by the Italian government to decide whether or not to introduce additional restrictive measures.

We assume that the finite set of mobility restriction combinations $\mathcal{U}$ corresponds to the different strategies applied by the Italian government to tackle the COVID-19 outbreak. We indicate these scenarios with different colors: white, green, yellow, orange, and red, respectively, corresponding to an increasing severity level of the applied restrictions. A detailed description of these scenarios can be found in Appendix A. In order to estimate the value of the control actions in the given scenarios, we make use of the Google Mobility Reports [25]. In fact, in our work, we assume that the infection rate is a function of the mobility level in different socio-economic categories. The Google Mobility Reports are divided into several categories; however, we select $G=3$ categories that are the most significant from the socio-economic perspective: the Workplaces, Retail \& recreation, and Public transport categories (see Appendix A for a detailed description). Accordingly, we define $\mathbf{m}(k)=\left(m_{1}(k), m_{2}(k), m_{3}(k)\right)^{\top}$, where $m_{1}(k), m_{2}(k)$, and $m_{3}(k)$ are the mobility level related to the Workplaces, Retail \& recreation, and Public transport categories at time step $k$, respectively. In particular, we assume that the different restrictions applied by the government lead to a reduction in the mobility; consequently, we compute the control actions at time step $k$ as $u_{g}(k)=1-m_{g}(k)$ for each category $g=1,2,3$, and thus we have $\mathbf{u}(k)=\left(u_{1}(k), u_{2}(k), u_{3}(k)\right)^{\top} \in \mathcal{U}$.

As a consequence, we estimate the value of the control action for the white, green, yellow, orange, and red scenario by averaging the control actions, computed employing the Google Mobility Reports from September 1 to December 5, 2020, in the periods when the different measures have been applied. In Table I, we show the estimated control actions with different restrictive scenarios. Note that for several regions, the control actions corresponding to a specific scenario is absent since the region was never under that scenario. In this case, we use the mean value for the overall country.

As for the objective function in (15), the cost coefficients are based on the percentage of the Italian gross domestic product correlated with the different categories and are thus set to
$0.15,0.80$, and 0.05 , i.e., $\phi=(0.15,0.8,0.05)^{\top}$. Note that the selection of these parameters is a choice of policy-makers; indeed, our approach allows administrators to adopt different governmental policies.

\section{B. The Proposed Stochastic Control Approach: Results and Discussion}

In order to simulate the spread of COVID-19 secondary waves under the effects of restrictive strategies, we divide the test bench into two different and independent components: the model of the COVID-19 dynamics and the control system.

The first part relies on the SIRCQTHE model to generate, at each time step, the new value for the different classes, i.e., what we consider as the real value in our simulation. However, to simulate the uncertainty and correctly evaluate the proposed stochastic framework, we include at each time step randomly generated disturbances on the number of people in each class with a maximum of $\pm 10 \%$ percentage variation. Moreover, at each $\omega$ time step we change the model parameters with a maximum of $\pm 20 \%$ in order to simulate a profound variation in the spreading scenario. However, to maintain the significance of the simulation we keep the parameters in the bound defined in Table II in Appendix A. Finally, it should be noted that the model of the COVID-19 dynamics is susceptible to the control system input, that is, the reduction of the people mobility caused by the application of the restrictive measures.

The second component, namely the control system, obtains, at each time step, the new data from the model of COVID-19 dynamics. However, it gets only the value of state variables that can be directly observed, i.e., the value of Quarantined, Threatened, Recovered, and Extinct individuals. In contrast, the value of the Susceptible, Infected, and Contagious people is unknown. Consequently, we assume that the control system estimates the SIRCQTHE model parameters every $\omega$ time steps through the Dynamical Identification Algorithm presented in Appendix A with a fitting window $\tau_{1}$ equal to 3 weeks.

Based on the estimated parameters for the SIRCQTHE model, the control system selects the most suitable strategy to apply by solving the optimal control problem. Therefore, let us first evaluate the forecasting system's prediction over the control horizon and the results of the stochastic optimization problem when $\alpha_{3}=1$ (i.e., the threshold for the Threatened cases is set to half the total number of beds.). In particular, we solve the stochastic optimal control problem by generating $M=1000$ IID samplings of the model parameters and assuming that the level of the risk that the decision-maker is willing to accept is $\epsilon=0.2$. The samplings are made employing truncated normal distributions with bounds defined in Table II and standard deviation equal to the $10 \%$ of the size of the interval between the two bounds. The results are the restrictive measures that the government should apply for the whole control horizon, highlighted with different background colors in Fig. 3. Having defined the control actions for the control horizon, a Monte Carlo simulation with 1,000 iterations is performed by randomly changing the model parameters at each time. Then, we calculate the expected value for the Threatened cases and its confidence interval, in the presence of the restriction measures determined by the Stochastic MPC. As shown 
TABLE I

SET OF THE ESTIMATED CONTROL ACTIONS RELATED TO THE SCENARIO CONSIDERED IN THE NUMERICAL EXPERIMENTS.

\begin{tabular}{|c|c|c|c|c|c|c|c|c|c|c|c|c|c|c|c|}
\hline Name & Retail & $\begin{array}{c}\text { White } \\
\text { Workplaces }\end{array}$ & Transport & Retail & $\begin{array}{c}\text { Green } \\
\text { Workplaces }\end{array}$ & Transport & Retail & $\begin{array}{c}\text { Yellow } \\
\text { Workplaces }\end{array}$ & Transport & Retail & $\begin{array}{r}\text { Orange } \\
\text { Workplaces }\end{array}$ & Transport & Retail & $\begin{array}{c}\text { Red } \\
\text { Workplaces }\end{array}$ & Transport \\
\hline Piedmont & 0.11 & 0.20 & 0.18 & 0.22 & 0.19 & \begin{tabular}{c|}
1 \\
0.25
\end{tabular} & - & - & \begin{tabular}{|c|c} 
& - \\
\end{tabular} & - & - & \begin{tabular}{l|l} 
\\
-
\end{tabular} & 0.55 & 0.34 & 0.54 \\
\hline Aosta & - & - & - & 0.40 & 0.22 & 0.40 & - & - & - & - & - & - & 0.69 & 0.39 & 0.63 \\
\hline Lombardy & 0.15 & 0.24 & 0.25 & 0.25 & 0.23 & 0.33 & - & - & - & - & - & - & 0.57 & 0.36 & 0.59 \\
\hline Trentino - S. Tyrol & 0.07 & 0.15 & 0.00 & 0.28 & 0.15 & 0.14 & 0.54 & 0.32 & 0.40 & - & - & - & - & - & \\
\hline Veneto & 0.06 & 0.17 & 0.13 & 0.17 & 0.14 & 0.21 & 0.30 & 0.22 & 0.42 & - & - & - & - & - & \\
\hline Friuli-Ven. Giulia & 0.07 & 0.17 & 0.05 & 0.17 & 0.15 & 0.10 & 0.29 & 0.21 & 0.27 & 0.49 & 0.26 & 0.36 & - & - & \\
\hline Liguria & 0.06 & 0.13 & 0.07 & 0.22 & 0.18 & 0.22 & 0.31 & 0.24 & 0.32 & 0.41 & 0.29 & 0.37 & - & - & \\
\hline Emilia-Romagna & 0.07 & 0.16 & 0.15 & 0.17 & 0.14 & 0.20 & 0.29 & 0.21 & 0.40 & 0.45 & 0.24 & 0.47 & - & - & \\
\hline Tuscany & 0.07 & 0.15 & 0.10 & 0.21 & 0.16 & 0.22 & 0.32 & 0.23 & 0.35 & 0.43 & 0.28 & 0.43 & 0.54 & 0.31 & 0.52 \\
\hline Umbria & 0.07 & 0.15 & 0.00 & 0.21 & 0.15 & 0.11 & 0.34 & 0.23 & 0.25 & 0.44 & 0.26 & 0.35 & - & - & \\
\hline Marche & 0.03 & 0.12 & 0.01 & 0.18 & 0.13 & 0.12 & 0.28 & 0.20 & 0.27 & 0.43 & 0.23 & 0.38 & - & - & \\
\hline Lazio & 0.15 & 0.25 & 0.31 & 0.24 & 0.24 & 0.36 & 0.32 & 0.29 & 0.47 & - & - & - & - & - & \\
\hline Abruzzo & 0.01 & 0.12 & 0.00 & 0.16 & 0.15 & 0.10 & 0.28 & 0.22 & 0.29 & 0.41 & 0.27 & 0.42 & 0.53 & 0.34 & 0.55 \\
\hline Molise & - & - & - & 0.15 & 0.11 & 0.06 & 0.31 & 0.24 & 0.34 & - & - & - & - & - & \\
\hline Campania & 0.07 & 0.22 & 0.14 & 0.26 & 0.26 & 0.31 & 0.37 & 0.33 & 0.45 & - & - & - & 0.55 & 0.40 & 0.59 \\
\hline Apulia & 0.00 & 0.16 & 0.04 & 0.15 & 0.16 & 0.20 & - & - & - & 0.39 & 0.29 & 0.49 & - & - & \\
\hline Basilicata & 0.00 & 0.13 & - & 0.13 & 0.12 & 0.29 & 0.29 & 0.22 & 0.47 & 0.41 & 0.29 & 0.56 & - & - & \\
\hline Calabria & 0.00 & 0.12 & 0.00 & 0.17 & 0.16 & 0.10 & - & - & - & - & - & - & 0.52 & 0.38 & 0.51 \\
\hline Sicily & 0.04 & 0.16 & 0.05 & 0.19 & 0.17 & 0.19 & - & - & - & 0.43 & 0.30 & 0.49 & - & - & \\
\hline Sardinia & 0.00 & 0.14 & 0.00 & 0.15 & 0.15 & 0.12 & 0.25 & 0.23 & 0.33 & - & - & - & - & - & \\
\hline Mean & 0.06 & 0.16 & 0.09 & 0.20 & 0.17 & 0.20 & 0.32 & 0.24 & 0.36 & 0.43 & 0.27 & 0.43 & 0.56 & 0.36 & 0.56 \\
\hline
\end{tabular}

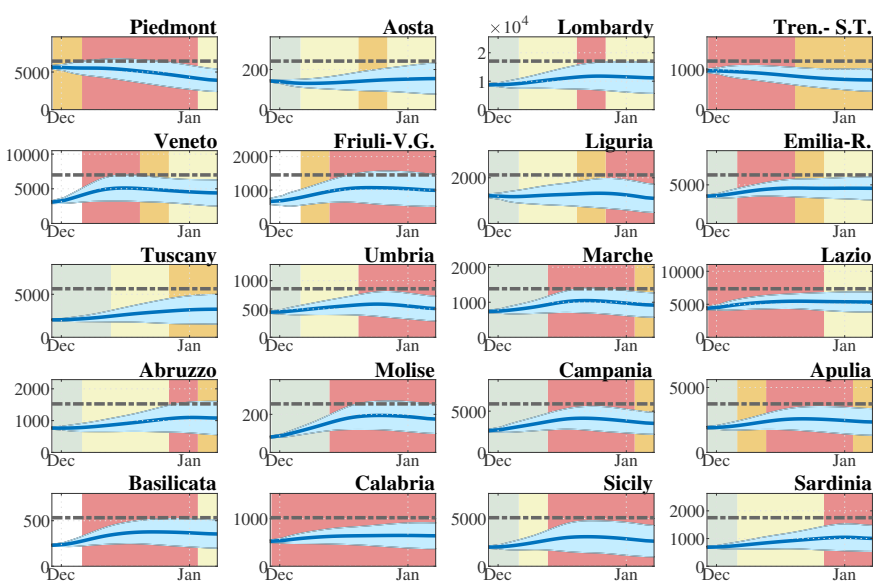

Fig. 3. Results of the Monte Carlo simulation in terms of the Threatened cases over the control horizon for each Italian region: expected Threatened cases (blue line), confidence interval for the Threatened cases (cyan area), maximum number of Threatened cases for each region (black dotted line), and the evolution of the control action in the control horizon (different background colors). For each region, the $\mathrm{x}$-axis reports times in days and the $\mathrm{y}$-axis represents the number of Threatened individuals.

in Fig. 3, where the blue line represents the time evolution of the expected Threatened cases, the cyan area represents its confidence interval, and the black line represents the maximum number of Threatened cases that can be treated by each Italian region, it is apparent that the proposed approach is able to keep the average number of cases under the maximum level.

In the optimal control problem (13), we include two penalizing factors: $\alpha_{1}$ smooths the optimal control action avoiding bang-bang controls, whilst $\alpha_{2}$ reduces the number of times that a change of decision taken in the previous time step occurs. However, the selection of these parameters should be made also taking into account other aspects that lie outside the mere technical aspects. Therefore, let us analyze the impact these two parameters have on reducing the control action switches. Let us preliminarily define by $\Delta s$ the total number of switches occurring in the current control action over the simulation period and by $\Delta m$ the total number of switches that the current control action shows with respect to the previous time step decision over the simulation period. We analyze the variation of these two kinds of switches with respect to the independent variation of $\alpha_{1}$ and $\alpha_{2}$ by varying one of the two parameters keeping the other equal to zero. We summarize the results of this analysis in Fig. 4 (a)-(b), where it is clear that by increasing these parameters, the number of switches decreases until an asymptotic value.

However, the selection of the best performing control actions is not the only aspect to be considered. Various aspects increase the complexity of the decision-making process. For instance, the selection of a proper threshold value is nontrivial; in the previous experiments, we set $\alpha_{3}=1$. However, this value profoundly influences other aspects that must be quantified to identify the gap between the best performing control actions and the others. Comparing the different control actions from various individual points of view can help policy-makers deeply analyze and choose the most effective action. Therefore, in Fig. 4 (c)-(d) we show the total number of Extinct people in the simulation period and the economic cost with respect to parameter $\alpha_{3}$. As expected, the number of deaths increases with this value, while the economic cost decreases. Moreover, in the last sensitivity analysis, we estimate the impact of parameter $\epsilon \in[0,1]$ which describes the risk level that the decision-maker is willing to accept when selecting the best control action to apply. In fact, as we employ a chance constraints approach we aim to satisfy the safety threshold in a probabilistic way. Hence, in Fig. 4 (e)-(f) we show the total number of Extinct people in the simulation period and the economic cost by changing parameter $\epsilon$. Obviously, increasing the risk level the percentage of fatalities will increases while the economic cost will decreases.

Let us now show the results of our approach in a rolling horizon fashion over a simulation period of 10 weeks, and let us analyze in detail the proposed framework by setting $\alpha_{3}=0.5$. Figure 5 shows the results obtained for two different set of values of parameters $\alpha_{1}$ and $\alpha_{2}$. In particular, Fig. 5 (a) 
(a)

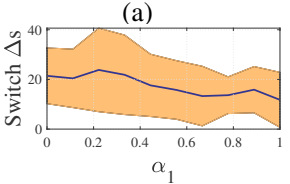

(d)

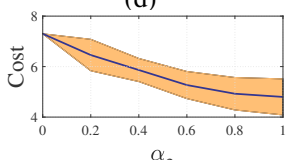

$\alpha_{3}$ (b)

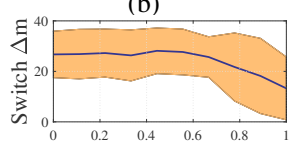

$\alpha_{2}$

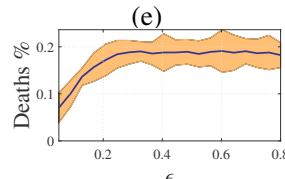

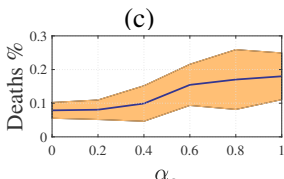

$\alpha_{3}$

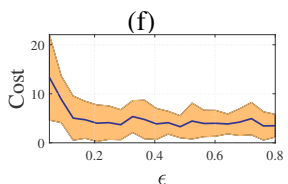

Fig. 4. Analysis of the results over the simulation period with respect to tuning parameters: total number of switches $\Delta s$ as a function of parameter $\alpha_{1}$ (a), total number of switches $\Delta m$ as a function of parameter $\alpha_{2}$ (b), total number of deaths as a function of parameter $\alpha_{3}$ (c), total economic cost as a function of parameter $\alpha_{3}$ (d), total number of deaths as a function of parameter $\epsilon(\mathrm{e})$ and total economic cost as a function of parameter $\epsilon(\mathrm{f})$.

is related to $\alpha_{1}=\alpha_{2}=0.1$, while in Fig. 5 (b) we set $\alpha_{1}=\alpha_{2}=1$. In both Figs. 5 (a)-(b), the blue line represents the time evolution of the Threatened cases, the black line represents the maximum number of Threatened cases that can be treated by each region, while the different background colors represent the evolution of the control action applied in different weeks. The simulations show that, by employing higher values of $\alpha_{1}$ and $\alpha_{2}$, it is possible to smooth the control action, while keeping the number of cases lower, albeit with an increased socio-economic cost. This comparative analysis is beneficial for policy-makers when suitable restrictive measures must be enforced. In fact, selecting the most beneficial strategy is not straightforward, since various factors increase the complexity of the decision-making context.

Summing up, the above simulations show that the proposed optimal control approach is able to keep the number of Threatened cases below a maximum limit, while minimizing the economic cost of the eventually required restriction periods. Specifically, selecting a suitable threshold on the maximum number of Threatened cases may profoundly influence secondary outcomes for the proposed approach, with obvious significance for policy-makers.

\section{Conclusions And Future Works}

Over the last months the COVID-19 pandemic has affected the whole world. Since the just started vaccinations require a long time to obtain herd immunity, the main control actions rely on the use of non-pharmaceutical interventions, such as the use of face masks and social distancing. Such measures, in conjunction with partial or total lockdown strategies, have been effective in mitigating the disease propagation, albeit at the cost of a significant socio-economic impact on the population. Nonetheless, as far as these measures have been relaxed, contagion has started to spread again, leading to secondary waves in many countries worldwide.

It is therefore essential to develop suitable methods to support policy-makers in efficiently mitigating the effects of COVID-19 pandemic contagions. To this aim, this paper presents a novel feedback control strategy which makes joint use of an epidemiological SIR-based model aimed at modeling the system dynamics and predicting its evolution in conjunction with a non-linear stochastic Model Predictive Control
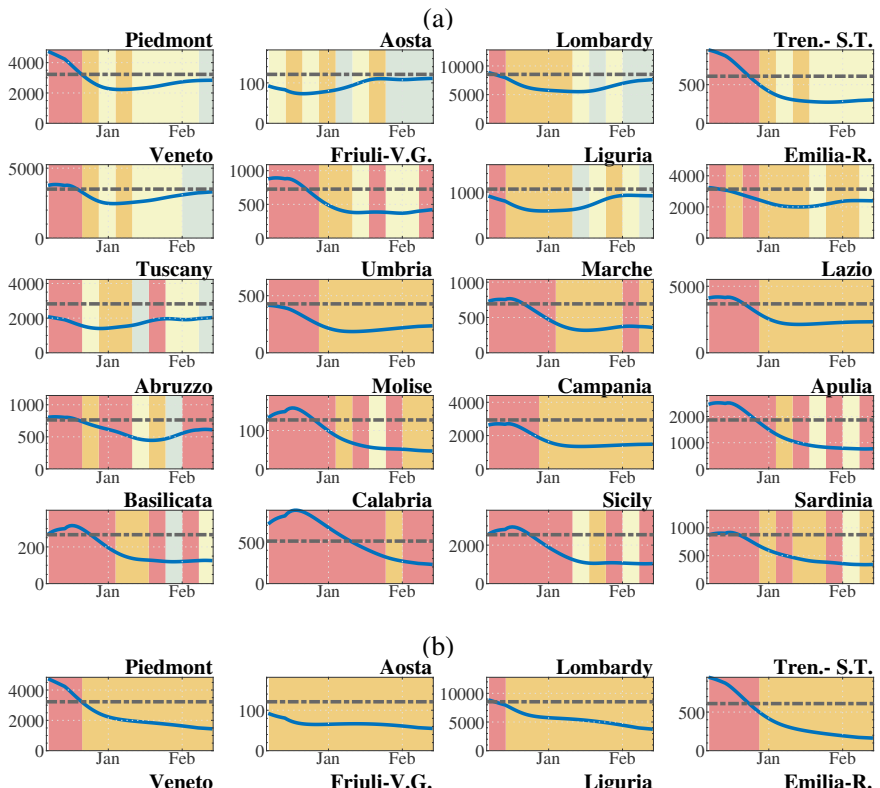

(b)
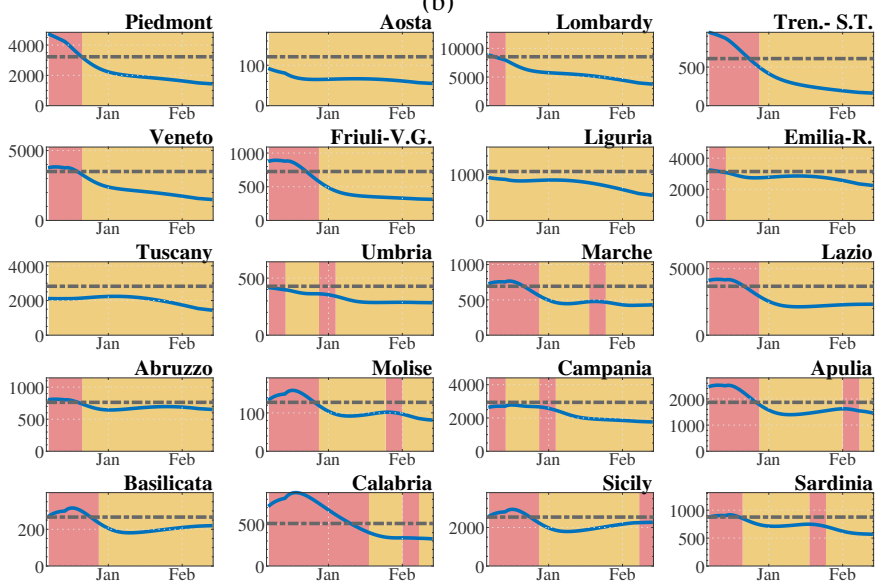

Fig. 5. Results obtained by the stochastic MPC for each region when $\alpha_{1}=$ $\alpha_{2}=0.1$ and $\alpha_{3}=0.5$ (a) and $\alpha_{1}=\alpha_{2}=1, \alpha_{3}=0.5$ (b): Threatened cases (blue line), maximum number of Threatened cases that can be treated by each region (black line), and evolution of the control action in the control horizon (different background colors). For each region, the $\mathrm{x}$-axis reports times in days and the $y$-axis represents the number of Threatened individuals.

(MPC) aimed at identifying the most effective control actions to tackle the contagions. More in detail, the epidemiological model consists in a novel time-varying SIRCQTHE model, so that eight different compartments of individuals and the connections between them are modeled to ensure a detailed representation of the pandemic dynamics: Susceptible, Infected, Removed, Contagious, Quarantined, Threatened, Healed, and Extinct.

The model also employs a multi-region framework, to properly represent the regional differences in healthcare systems, thus also allowing to deepen the prediction while at the same time ensuring that the subsequent predictive controller provides the mitigation of contagions at each regional level. Since the infection rate depends on people's mobility, the control actions are modeled as interventions affecting the mobility levels associated to different socio-economic categories. The optimal control approach thus aims at minimizing the socioeconomic cost of the mobility reduction, that is, the impact of the adopted restrictive measures, and, thanks to its stochastic formulation, also ensures a robust solution of the problem (e.g., in case of possible errors when estimating the variables or in the presence of disturbances on the model). Finally, 
feedback control ensures that the predicted future output of the model are kept in line with the selected target objective.

The proposed approach is tested on the real data of the Italian scenario, which is composed by 20 regions with separated administrative bodies cooperating with the central national government, and presenting relevant differences in the healthcare systems. The considered case study shows the effectiveness of the presented method in tackling the problem by keeping the number of Threatened cases below a fixed maximum limit, while minimizing the socio-economic impact of the required restriction periods. Thanks to its generalizability, the presented method fills the gaps encountered in the relevant literature representing an effective tool to control the COVID19 pandemic.

Future research will address integrating additional objective functions and constraints in the optimal control problem, Moreover, we will use other type of models and machine learning techniques to identify the model's characteristic parameters, such as estimating the relation between the infection rate and the mobility level. As a further development of the approach, age-differentiated control actions can also be addressed, and, with the ongoing vaccination for the COVID19 disease, we also plan to investigate the most effective way for distribution of vaccine to population.

\section{APPENDIX A}

\section{PARAMETERS IDENTIFICATION}

Several epidemiological models have been proposed in the literature for modeling the dynamics of COVID-19; however, the quantity and quality of measured data makes challenging the calibration of the related parameters.

Therefore, in this appendix, we explain how we calibrate the SIRCQTHE model to the Italian scenario for the considered numerical experiments. We divide the appendix into four parts. In the first part, we explain the Italian epidemiological scenario in the COVID-19 second wave of contagions, together with the Italian government's response actions and strategies adopted to tackle the outbreak. In the second part, we describe the raw data available for the Italian scenario and the main assumptions on the system parameters. In the third part, we present the different step of the fitting procedure, while in the last part, we present the algorithm for the dynamical fitting of the SIRCQTHE model used in the predictive control framework.

\section{A. Description of the Italian Scenario}

After being one of the most affected nations in the first stage of the COVID-19 outbreak, thanks to a two-months lockdown, in Italy the pandemic started to decline in May 2020. Consequently, inter- and intra-region mobility was re-established on May 4, while other not essential activities were re-opened later in the same month. After a relatively stable situation during the summer, a rise of contagions has been experienced from September 2020. Consequently, renewed restrictions have been progressively introduced (decree-laws of October 7, 13, 18 , and 24 2020). Moreover, after the exponential growth of cases, from November 62020 , the Italian regions have been grouped into three different epidemiological categories, identified by the yellow, orange, and red colors that include different restrictive measures [35]. Nevertheless, this division is not static, since a region can change its color due to a modified epidemiological scenario.

Hereafter, we describe the restrictive measures adopted by the Italian Government grouped by period of adoption and by type of restrictions. For a matter of clarity we indicate each group by a different color.

White: In this class, we include all the restrictions adopted by the Italian government at a national level from the end of the emergency (namely June 15) to October 8. Almost all the restrictions are withdrawn, except for the closure of discos and nightclubs (not in all regions). The number of seats in cinemas and theaters is limited, and the use of masks is mandatory.

Green: In this class, we include all the actions taken at a national level as the number of cases in November rapidly increased. The maximum number of people allowed in parties and ceremonies is limited. Moreover, congresses and festivals are suspended. Other measures are adopted with the closure of gyms, swimming pools, theaters, and cinemas. Lastly, the government imposes the closure at 6.00 p.m. of restaurants and bars and the prohibition of parties.

Yellow: In this class, all the restrictions of the green class are considered. Moreover, a curfew from 10:00 p.m. to 5:00 a.m. is introduced (unless justified reasons for work, emergencies, and health). Shopping centers are closed on holidays. Teleeducation for high schools is introduced. Universities are also closed. For bars and restaurants, take-away is allowed until 10 p.m.

Orange: In this class, all restrictions of the previous strategies are considered. Moreover, it is forbidden to travel between regions and between municipalities unless proven reasons. Bars and restaurants are closed while the take-away service is allowed until 10 p.m.

Red: In this class, all the restrictions of the previous strategies are considered. Moreover, it is forbidden to move also inside a municipality without a justified reason. Stores are closed except for supermarkets. Newsstands, tobacco stores, pharmacies, hairdressers, and barbers shop remain open; nevertheless, beauty centers are closed. Distance education is provided for high school and third-grade classes in middle school. Almost all sports competitions are suspended while sports activity is allowed only near home in individual form.

\section{B. Data Availability for the Italian Scenario and Main As- sumptions on the SIRCQTHE Model Parameters}

The Italian Civil Protection Department (Protezione Civile) is the state body responsible to collect and elaborate all the data related to the pandemic. As soon as the very first cases of COVID-19 affected individuals were confirmed, the Protezione Civile department made available several epidemiological data [23].

The main problem related to these data, as well as to almost all the data similarly available worldwide, is that they represent a screenshot of the epidemiological situation each day. For each class, there is only available the number of people each day without any indication about the flows within the different 
categories. For instance, in the deceased category, it is not indicated the percentage of previously hospitalized people. This problem requires additional assumptions on the model and its parameters.

Most data are not updated regularly; e.g., some may not be updated on weekends. Therefore, the available data are affected by uncertainties and as such can lead to an imprecise identification of the model parameters. To overcome this limitation, we pre-process the data with a finite impulse response filter. In particular, we perform a fourteen-days weighted moving average with linearly decreasing weights in order to smooth the impact of outliers and irregular data on the identification process.

Besides the effects of uncertain data on the identification process, it is fundamental to consider the time-dependency of the parameters to perform a realistic and fruitful selection of the model parameters, especially on a long analysis period. In the related literature, a common approach adopted in order to overcome this problem consists of fitting the model with different time windows, following the stages of the pandemic [16]. This leads to a good fitting only when additional knowledge is introduced. Conversely, in our work, we fit the proposed SIRCQTHE model by assuming that some parameters can be correlated with proper time-dependent functions.

Hereafter, we detail the assumptions considered for each of the model parameters.

1) $\beta(k)$ : The so-called infection rate $\beta(k)$ for the COVID19 pandemic (see, e.g., the work in [8]) is usually assumed within 0.25 and 0.8 in the absence of any social distancing policies and people awareness. Nevertheless, lockdown periods can profoundly reduce this parameter. The use of different coefficients for each region helps the model fitting since $\beta$ is likely to increase in the regions with a higher population density (we refer the interested reader to [36]). In our work, in order to achieve a continuous fitting of the COVID-19 evolution in the second outbreak, we assume that the evolution of $\beta$ is related to the evolution of people's mobility estimated through the Google Mobility Reports [25]. The Google mobility reports show how visits and length of stay at different places change compared to a baseline. Changes for each day are compared to a baseline value for that day of the week. This dataset is based on data from users who have turned on the location history for their account; hence, the data represent a sample of all Google users. In particular, the mobility trends are divided into different categories.

We select the Workplaces, Retail \& recreation, and Public transport categories as the most significant in terms of socio-economic importance. We define $\mathbf{m}(k)=$ $\left(m_{1}(k), m_{2}(k), m_{3}(k)\right)^{\top}$, indicating respectively the mobility level of Workplaces, Retail and recreation and Public transport. Accordingly we set $\boldsymbol{\beta}=\left(\beta_{1}, \beta_{2}, \beta_{3}\right)^{\top}$. Hence, the infection rate is estimated as in (9).

2) $\rho$ : The so-called incubation rate $\rho$ is the rate of infected people that become contagious; consequently, they can infect other people. In the literature, the incubation time is estimated between 2 and 7 days [37]-[40].

3) $\gamma$ : The healing rate of unrecognized Contagious people $\gamma$ can be approximated by a constant since there is no proof that the virus has mutated. In particular, the literature findings show that the healing time is approximately 14 days. However, in our model, we do not remove people from the Contagious compartment when they are completely healed, but when they are not contagious or have a really low viral load. In the literature, this period is estimated between 3 and 10 days [28], [41], [42].

4) $\delta$ : The healing rate of Quarantined people who do not need hospitalization $\delta$ can also be approximated by a constant value. Moreover, $\delta$ can be substituted with $\gamma$ when a person is removed from the Quarantined compartment immediately after he/she becomes not contagious. However, in several countries, someone may be forced to be in quarantine even after clinically healed because the procedure requires two negative tests. In particular, in Italy, the quarantine period cannot be shorter than 10-14 days, depending on the swabs' tests results [24]. Nevertheless, due to the national health system's overload and the delay of swabs processing, this value may considerably increase [43]. Thanks to the structure of the SIRCQTHE model the healing rate $\delta$ can be expressed as follows:

$$
\delta=(H(k)-H(k-1)) / Q(k) .
$$

5) $\theta(k)$ : The parameter $\theta(k)$ models the rate of Contagious people recognized and Quarantined, the so-called detection rate. This is mainly related to the specific policy adopted by each region and the number of laboratory testing capacities (e.g., in terms of tested swabs). However, when laboratory limits are reached, the number of tested swabs becomes constant. Numerous researches point out that the ratio between new daily discovered cases $p(k)$ and daily swabs $w(k)$ is crucial to understand how the tracking system is operating. When this ratio increases, it means that the tracking system is not working well and that the pandemic is out of control, i.e., the real number of infected people is much higher than the recognized cases. Conversely, when this number is low, it means that few cases have been recognized with respect to the total amount of analyzed swabs; therefore, the pandemic is under control. Note that the aforementioned assumptions only hold when the tests are made in a stochastic way. In fact, if a country has a well-organized tracking system, it can perform only the necessary tests on people surely infected. Therefore, to consider the variability of the parameter $\theta(k)$, we relate it with the aforementioned ratio as:

$$
\theta(k)=\theta_{0}(1-r(k))
$$

where $r(k)=p(k) / w(k)$.

6) $\lambda$ and $\mu$ : These parameters are the hospitalization rates (i.e., the rate of people recognized only when severe symptomatic conditions occur and the rate of Quarantined people to be hospitalized, respectively). We assume these two parameters as constant because they are correlated only to the virus's characteristics.

7) $\pi$ : The recovery rate $\pi$ during the first outbreak of COVID-19 was far from being constant; in fact, the national healthcare system was not prepared and did not have healing procedures. However, after the implementation of new standardized clinical approaches, we assume that this parameter is constant. 
TABLE II

SIRCQTHE MODEL PARAMETER BOUNDS

\begin{tabular}{c|ll|l|l} 
& Description & & Bound & Reference \\
\hline$\beta_{0}$ & Base infection rate & $S \rightarrow I$ & $0.01-0.1$ & {$[8],[11],[43],[46]$} \\
$\beta_{g}$ & Mobility coefficients & $S \rightarrow I$ & $0.1-0.5$ & {$[8],[11],[43],[46]$} \\
$\rho$ & Incubation rate & $I \rightarrow C$ & $0.15-0.5$ & {$[37]-[40]$} \\
$\theta_{0}$ & Detection rate & $C \rightarrow Q$ & $0.001-0.5$ & {$[8],[11]$} \\
$\gamma$ & Healing rate & $C \rightarrow R$ & $0.1-0.3$ & {$[8],[28],[41]$} \\
$\delta$ & Healing rate & $Q \rightarrow H$ & $0.01-0.1$ & {$[8],[11],[24],[43]$} \\
$\lambda$ & Threatening rate & $C \rightarrow T$ & $0.001-0,02$ & {$[3],[8],[11]$} \\
$\mu$ & Threatening rate & $Q \rightarrow T$ & $0.001-0,08$ & {$[3],[8]$} \\
$\pi$ & Healing rate & $T \rightarrow H$ & $0.01-0,2$ & {$[3],[8],[11],[47]$}
\end{tabular}

8) $\varepsilon(k)$ : The death rate $\varepsilon$ at the beginning of a new epidemic is not constant and hopefully decreases with time. This is mainly due to the availability of new clinical treatments. The death rate for each time step $k$ can be formulated as follows:

$$
\varepsilon(k)=(E(k)-E(k-1)) / T(k) .
$$

With the pandemic's ongoing, this parameter becomes easily identifiable, and it only depends on how much the healthcare system is under pressure.

\section{Long-term Identification of the Model Parameters}

As we analyze the case of the 20 Italian regions that are heterogeneous in terms of the health care system, size, population, economy, and demography method should focus on how to design a predictive model with a strong capability of learning big data. Therefore we estimate the relationships between real data and the SIRCQTHE model's characteristic parameters employing some of the most known machine learning techniques [44], [45]. In particular, let us adopt an approach based on a least-squares optimization technique combined with constraints to enforce the prior scientific knowledge of the COVID-19 pandemic. In detail, based on the aforementioned assumption and the main literature findings, we define specific bounds on each parameter summarized in Table II.

Due to the high non-linearity of the proposed model, the fitting is highly non-convex and, in general, challenging. Therefore, let us introduce a multi-step fitting procedure that considers at each step a smaller sub-model with fewer parameters to be estimated.

In the first stage, we analyze the following sub-model:

$$
\begin{aligned}
\tilde{S}(k+1) & =\tilde{S}(k)-\left(\beta_{0}+\boldsymbol{\beta}^{\top} \mathbf{m}(k)\right) \tilde{C}(k) \tilde{S}(k) / N \\
\tilde{I}(k+1) & =\tilde{I}(k)-\rho \tilde{I}(k)+\left(\beta_{0}+\boldsymbol{\beta}^{\top} \mathbf{m}(k)\right) \tilde{C}(k) \tilde{S}(k) / N \\
\tilde{R}(k+1) & =\tilde{R}(k)+\gamma \tilde{C}(k) \\
\tilde{C}(k+1) & =\tilde{C}(k)+\rho \tilde{I}(k)-\left(\gamma+\lambda+\theta_{0}(1-r(k))\right) \tilde{C}(k) \\
Z(k+1) & =Z(k)+\left(\lambda+\theta_{0}(1-r(k))\right) \tilde{C}(k)
\end{aligned}
$$

where $Z(k)=Q(k)+T(k)+H(k)+E(k)$ is the cumulative number of Infected people. It is clear that $\beta_{0}, \boldsymbol{\beta}, \rho, \gamma, \theta_{0}, \lambda$ and the initial conditions $\tilde{I}(0)$ and $\tilde{C}(0)$ are the only parameters to be estimated. The estimation of such parameters consists in minimizing the mean squared error (MSE) of the model with respect to the real data, which is defined as:

$$
\operatorname{MSE}\left(\Xi_{1}\right)=\frac{1}{K} \sum_{k=1}^{K}\left(\frac{\hat{Z}\left(\Xi_{1}, k\right)-Z(k)}{Z(k)}\right)^{2}
$$

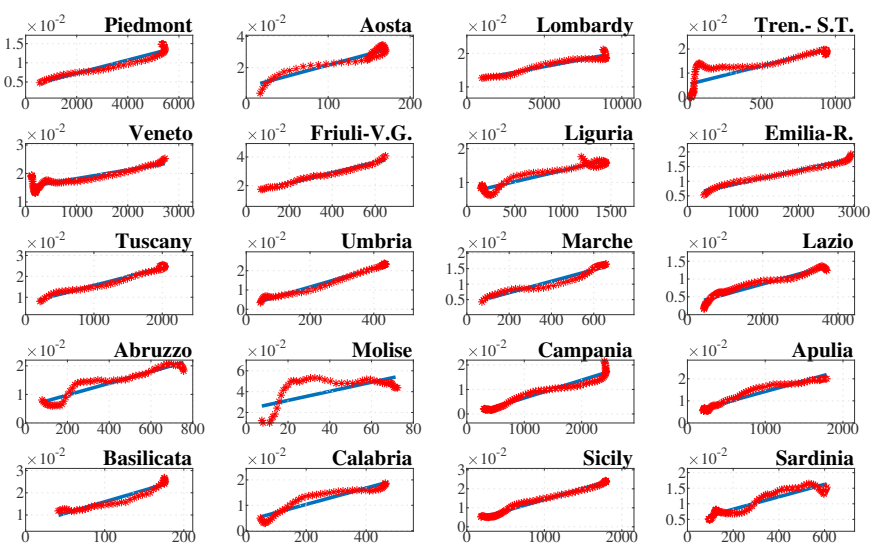

Fig. 6. Death rate with respect to the number of Threatened cases in all Italian regions: Real data (red asterisks) and fitting result (blue line).

where $\Xi_{1}=\left(\beta_{0}, \boldsymbol{\beta}, \rho, \gamma, \theta_{0}, \lambda, \tilde{I}(0), \tilde{C}(0)\right)$ collects the unknown parameters. Moreover, based on serological testing data [48] when searching for realistic parameters we assume that $\tilde{C}(k)<20(Q(k)+T(k))$.

In the second stage, we estimate the healing rate by:

$$
\delta=\frac{1}{K} \sum_{k=1}^{K}\left(\frac{H(k)-H(k-1)}{Q(k)}\right) .
$$

For the sake of space, we do not plot this rate over time; however, our findings show that the assumption made on its constancy is well based. We observe (excluding outliers) that this rate remained almost constant during the second wave. Nevertheless, we point out that this parameter is different in the north of Italy and southern regions. In the latter, this parameter is significantly lower. This can be explained with the poorer and less organized healthcare systems.

Moreover, as aforementioned we assume that the death rate follows a linear relation with respect to the number of hospitalized individuals $T(k)$ at time $k$. This relation is described by two characteristic parameters: the ordinate-axis intercept $\varepsilon_{0}$ and the gradient $\varepsilon_{1}$. The estimation of such coefficients consists in minimizing the mean squared error (MSE) of the linear approximation with respect to the real data, which is defined as:

$$
\operatorname{MSE}\left(\varepsilon_{0}, \varepsilon_{1}\right)=\frac{1}{K} \sum_{k=1}^{K}\left(\frac{\left(\varepsilon_{0}+\varepsilon_{1} T(k)\right)-\varepsilon(k)}{\varepsilon(k)}\right)^{2} .
$$

The correlation between the death rate and the number of Threatened people is evident in Fig. 6, where we show the Italian regions' death rate with respect to the number of Threatened cases computed employing the data from September to December 2020. The relation is evident except for the Molise region, where the tiny number of cases harms a profitable identification.

Having computed the aforementioned parameters, and having obtained an estimation of $\tilde{C}(k)$, we analyze the following sub-model:

$$
\begin{aligned}
Q(k+1)=Q(k)+\left(\theta_{0}(1-r(K))\right) & \tilde{C}(k) \\
& +\pi T(k)-(\delta+\mu) Q(k)
\end{aligned}
$$



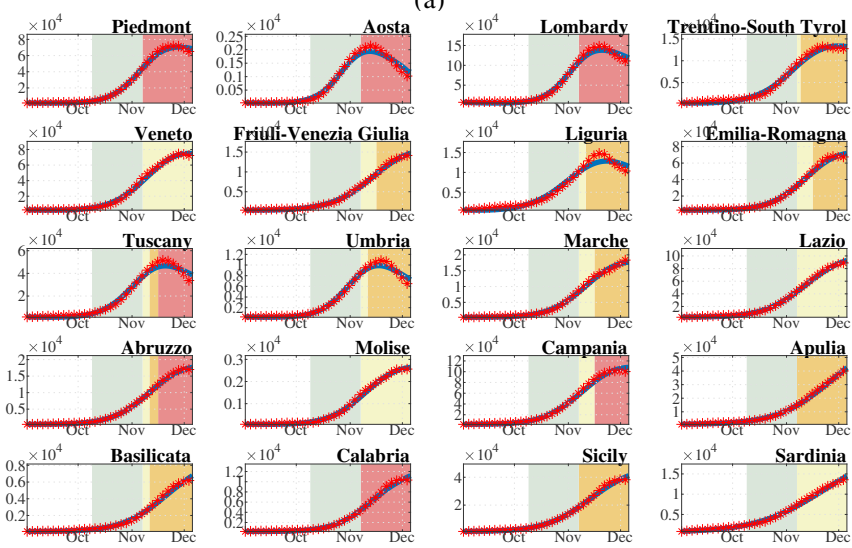

(c)
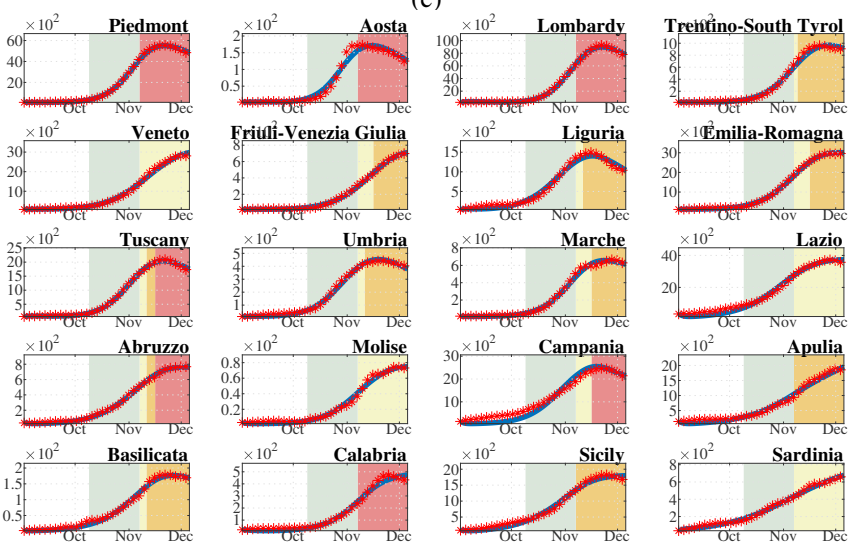
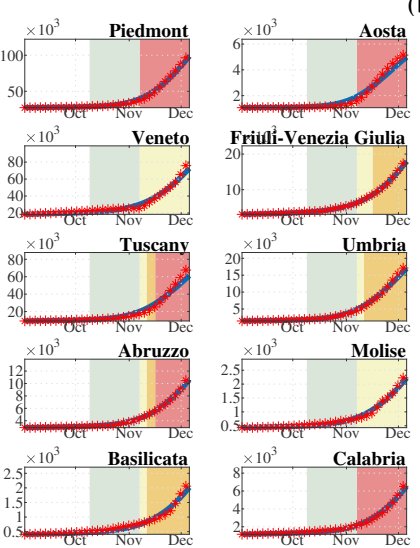

(b)
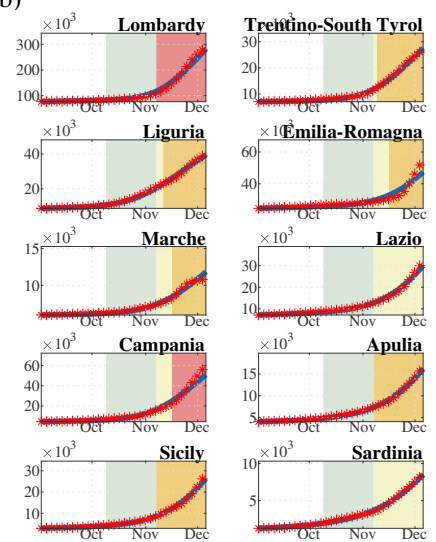

Fig. 7. Results of the fitting procedure for all the Italian regions: Quarantined (a), Healed (b), Threatened (c), and Deaths (d). Real data (red asterisks) and SIRCQTHE model output (blue line) over the evolution of restriction measures (different background colors).

$$
\begin{aligned}
& T(k+1)=T(k)+\mu Q(k)+\lambda \tilde{C}(k)-(\pi+\varepsilon(T(k))) T(k) \\
& H(k+1)=H(k)+\delta Q(k)
\end{aligned}
$$

and we minimize the following MSE:

$$
\begin{aligned}
& \operatorname{MSE}\left(\Xi_{2}\right)=\frac{1}{K} \sum_{k=1}^{K}\left(\frac{\hat{Q}\left(\Xi_{2}, k\right)-Q(k)}{Q(k)}\right)^{2} \\
& +\left(\frac{\hat{T}\left(\Xi_{2}, k\right)-T(k)}{T(k)}\right)^{2}+\left(\frac{\hat{H}\left(\Xi_{2}, k\right)-H(k)}{H(k)}\right)^{2}
\end{aligned}
$$

where $\Xi_{2}=\left(\mu, \pi, \theta_{0}, \lambda\right)$. Note that for the sake of a better fitting we estimate again the parameters $\theta_{0}$ and $\lambda$.

In order to improve the overall fitting procedure, an additional fitting procedure can be done considering the overall SIRCQTHE model employing as the initial guess for the optimizer the parameters computed with the sub-model procedure and using a $\pm 5 \%$ bound around the guess.

Figure 7 shows the real data and the outputs of the fitting procedure for compartments $Q, T, H$, and $E$, hence proving the effectiveness of the proposed procedure.

\section{Dynamical Identification of the Model Parameters}

In the previous section, we present a procedure that allows us to fit the SIRCQTHE model over a long period. However, since we aim to introduce this identification procedure in a rolling horizon fashion, let us present the Dynamical Identification (DI) Algorithm.

The definition of the algorithm is reported in Algorithm 1 and is summarized in the sequel. The algorithm runs at each time step $d$ and requires two integers: $\tau_{1}$ that indicates the fitting window and $\tau_{2}$ that is the forecasting period. Consequently, the algorithm requires in input the data related to the observable classes $Q\left(k-\tau_{1}: k\right), T\left(k-\tau_{1}: k\right)$, $H\left(k-\tau_{1}: k\right), E\left(k-\tau_{1}: k\right)$, and the data related to the mobility $\mathbf{m}\left(k-\tau_{1}: k\right)$, and the swabs' ratio $r\left(k-\tau_{1}: k\right)$.

In the first phase, all the time series are pre-processed with a weighted moving average filter, while all zeros and $\mathrm{NaN}$ (i.e., Not a Number) values are substituted. In the second phase, we compute the first set of model parameters $\beta_{0}, \beta_{1}, \beta_{2}, \beta_{3}, \rho, \gamma, \theta_{0}, \lambda \tilde{I}(0)$, and $\tilde{C}(0)$ based on fixed bounds given by the literature knowledge. Furthermore, we calculate the healing rate $\delta$ and and the coefficients $\varepsilon_{0}$ and $\varepsilon_{1}$ of the death rate linear relation. Next, we compute once again $\theta_{0}$ and $\lambda$ with the last parameters $\mu$ and $\pi$. After having all the parameters, we extrapolate a trend from the mobility value and the swabs ratio, and we estimate a value for the subsequent $\tau_{2}$ time steps. Lastly, we simulate the SIRCQTHE model with the fitted parameters and the estimated mobility and swabs ratio values over the $\tau_{2}$ time steps.

Note that, due to the lack of space, we do not show the results of the DI Algorithm and the fitted value for the Italian 


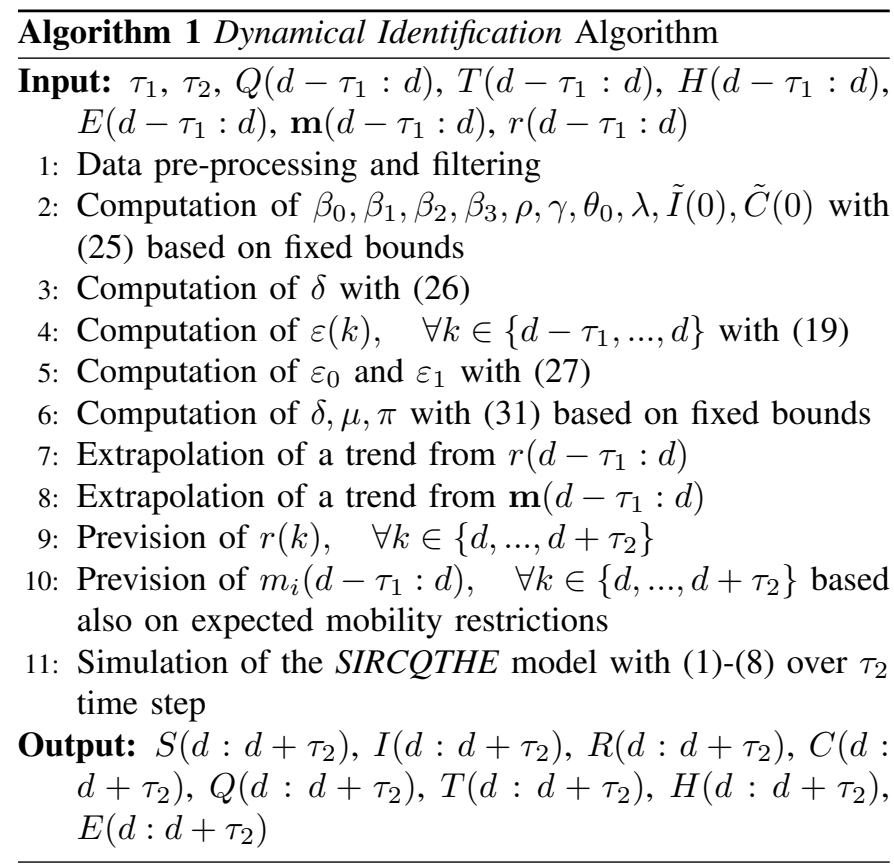

regions. The interested readers is referred to our website [49] where the fitted parameters are reported for all the regions and the forecasting (in different time scale) is shown in detail for the Apulian region.

\section{REFERENCES}

[1] "World Health Organization," https://www.who.int/emergencies/ diseases/novel-coronavirus-2019, [Accessed: 2020-12-29].

[2] N. Ferguson, D. Laydon, G. Nedjati-Gilani, N. Imai, et al., "Report 9: Impact of non-pharmaceutical interventions (npis) to reduce covid19 mortality and healthcare demand," Imperial College London, vol. 10, p. $77482,2020$.

[3] G. Giordano, F. Blanchini, R. Bruno, P. Colaneri, et al., "Modelling the COVID-19 epidemic and implementation of population-wide interventions in Italy," Nat. Med., vol. 26, p. 855-860, 2020.

[4] R. Carli, G. Cavone, N. Epicoco, P. Scarabaggio, and M. Dotoli, "Model predictive control to mitigate the COVID-19 outbreak in a multi-region scenario," Аnnu. Rev. Control, vol. 50, pp. 373 - 393, 2020.

[5] C. Nowzari, V. M. Preciado, and G. J. Pappas, "Analysis and control of epidemics: A survey of spreading processes on complex networks," IEEE Control Syst. Mag., vol. 36, no. 1, pp. 26-46, 2016.

[6] P. E. Paré, C. L. Beck, and T. Başar, "Modeling, estimation, and analysis of epidemics over networks: An overview," Annu. Rev. Control, vol. 50, pp. $345-360,2020$.

[7] C. T. Deressa and G. F. Duressa, "Modeling and optimal control analysis of transmission dynamics of covid-19: The case of ethiopia," Alex. Eng. J., vol. 60, no. 1, pp. 719 - 732, 2021.

[8] F. Della Rossa, D. Salzano, A. Di Meglio, F. De Lellis, et al., "A network model of italy shows that intermittent regional strategies can alleviate the covid-19 epidemic," Nat. Commun., vol. 11, p. 5106, 2020.

[9] E. Scharbarg, C. H. Moog, N. Mauduit, and C. Califano, "From the hospital scale to nationwide: observability and identification of models for the covid-19 epidemic waves," Annu. Rev. Control, vol. 50, pp. 409 $-416,2020$.

[10] S. Ansumali, S. Kaushal, A. Kumar, M. K. Prakash, and M. Vidyasagar, "Modelling a pandemic with asymptomatic patients, impact of lockdown and herd immunity, with applications to SARS-CoV-2," Annu. Rev. Control, vol. 50, pp. 432 - 447, 2020.

[11] M. Gatto, E. Bertuzzo, L. Mari, S. Miccoli, et al., "Spread and dynamics of the covid-19 epidemic in italy: Effects of emergency containment measures," Proc. Nat. Acad. Sci., vol. 117, no. 19, pp. 10484-10491, 2020.

[12] L. Di Domenico, G. Pullano, P. Coletti, N. Hens, and V. Colizza, "Expected impact of school closure and telework to mitigate covid-19 epidemic in france," Report, Tech. Rep., 2020.
[13] L. Brugnano, F. Iavernaro, and P. Zanzottera, "A multiregional extension of the SIR model, with application to the COVID-19 spread in Italy," Math. Method. Appl. Sci., 2020.

[14] J. Köhler, L. Schwenkel, A. Koch, J. Berberich, P. Pauli, and F. Allgöwer, "Robust and optimal predictive control of the COVID-19 outbreak," Annu. Rev. Control, 2020.

[15] M. M. Morato, S. B. Bastos, D. O. Cajueiro, and J. E. Normey-Rico, "An optimal predictive control strategy for COVID-19 (SARS-CoV-2) social distancing policies in Brazil," Annu. Rev. Control, vol. 50, pp. $417-431,2020$.

[16] M. Bin, P. Y. Cheung, E. Crisostomi, P. Ferraro, H. Lhachemi, R. Murray-Smith, C. Myant, T. Parisini, R. Shorten, S. Stein, et al., "Post-lockdown abatement of COVID-19 by fast periodic switching," PLoS computational biology, vol. 17, no. 1, p. e1008604, 2021.

[17] E. H. Bussell, C. E. Dangerfield, C. A. Gilligan, and N. J. Cunniffe, "Applying optimal control theory to complex epidemiological models to inform real-world disease management," Philos. Trans. R. Soc. B, vol. 374, no. 1776, p. 20180284, 2019.

[18] F. Sélley, Á. Besenyei, I. Z. Kiss, and P. L. Simon, "Dynamic control of modern, network-based epidemic models," SIAM J. Appl. Dyn. Syst., vol. 14, no. 1, pp. 168-187, 2015.

[19] G. Franzè, F. Tedesco, and D. Famularo, "Resilience against replay attacks: A distributed model predictive control scheme for networked multi-agent systems," IEEE/CAA Journal of Automatica Sinica, vol. 8, no. 3, pp. 628-640, 2020.

[20] Y. Cao and J. Huang, "Neural-network-based nonlinear model predictive tracking control of a pneumatic muscle actuator-driven exoskeleton," IEEE/CAA Journal of Automatica Sinica, vol. 7, no. 6, pp. 1478-1488, 2020.

[21] C. Tsay, F. Lejarza, M. A. Stadtherr, and M. Baldea, "Modeling, state estimation, and optimal control for the US COVID-19 outbreak," Nat. Sci. Rep., vol. 10, p. 10711, 2020.

[22] S. Grundel, S. Heyder, T. Hotz, T. K. S. Ritschel, P. Sauerteig, and $\mathrm{K}$. Worthmann, "How much testing and social distancing is required to control COVID-19? some insight based on an age-differentiated compartmental model," arXiv preprint arXiv: 2011.01282v1, 2020.

[23] "The Civil Protection Department COVID-19 dashboard," http://opendatadpc.maps.arcgis.com/apps/opsdashboard/index.html\# /b0c68bce2cce478eaac82fe38d4138b1, [Accessed: 2020-12-07].

[24] "Covid-19: indicazioni per la durata e il termine dell'isolamento e della quarantena," http://www.salute.gov.it/portale/nuovocoronavirus/ dettaglioNotizieNuovoCoronavirus.jsp, [Accessed: 2020-12-07].

[25] "Google COVID-19 Community Mobility Reports," www.google.com/ covid19/mobility, [Accessed: 2020-12-07].

[26] C. Santamaria, F. Sermi, S. Spyratos, S. M. Iacus, et al., "Measuring the impact of covid-19 confinement measures on human mobility using mobile positioning data. a european regional analysis," Saf. Sci., vol. 132, p. 104925, 2020.

[27] L. Guan, C. Prieur, L. Zhang, C. Prieur, D. Georges, and P. Bellemain, "Transport effect of covid-19 pandemic in france," Аnnu. Rev. Control, 2020.

[28] Y. Bai, L. Yao, T. Wei, F. Tian, et al., "Presumed asymptomatic carrier transmission of covid-19," JAMA, vol. 323, no. 14, pp. 1406-1407, 2020.

[29] X. Liu and X. Kong, "Nonlinear model predictive control for dfig-based wind power generation," IEEE Trans. Autom. Sci. Eng., vol. 11, no. 4 , pp. 1046-1055, 2013.

[30] S. M. Iacus, C. Santamaria, F. Sermi, S. Spyratos, D. Tarchi, and M. Vespe, "Human mobility and covid-19 initial dynamics," Nonlinear Dynamics, vol. 101, no. 3, pp. 1901-1919, 2020.

[31] G. Tian, M. Zhou, and J. Chu, "A chance constrained programming approach to determine the optimal disassembly sequence," IEEE Trans. Autom. Sci. Eng., vol. 10, no. 4, pp. 1004-1013, 2013.

[32] MATLAB, 9.8.0.135996 (R2020b). The MathWorks Inc., 2020.

[33] H. C. Lau, T. Chan, W. Tsui, and W. Pang, "Application of genetic algorithms to solve the multidepot vehicle routing problem," IEEE Trans. Autom. Sci. Eng., vol. 7, no. 2, pp. 383-392, 2009.

[34] "Portale Covid-19," www.agenas.gov.it/covid19/, [Accessed: 2020-1207].

[35] "Dpcm del 3 novembre," http://www.governo.it/it/articolo/ dpcm-del-3-novembre-le-misure-suddivise-aree-di-criticit, [Accessed: 2020-12-07].

[36] H. Hu, K. Nigmatulina, and P. Eckhoff, "The scaling of contact rates with population density for the infectious disease models," Math. Biosci., vol. 244, no. 2, pp. 125-134, 2013.

[37] W.-J. Guan, Z.-Y. Ni, Y. Hu, W.-H. Liang, et al., "Clinical characteristics of coronavirus disease 2019 in china," N. Engl. J. Med., vol. 382, no. 18, pp. 1708-1720, 2020. 
[38] S. A. Lauer, K. H. Grantz, Q. Bi, F. K. Jones, et al., "The incubation period of coronavirus disease 2019 (covid-19) from publicly reported confirmed cases: estimation and application," Ann. Intern. Med., vol. 172 , no. 9 , pp. 577-582, 2020.

[39] Q. Li, X. Guan, P. Wu, X. Wang, et al., "Early transmission dynamics in wuhan, china, of novel coronavirus-infected pneumonia," N. Engl. J. Med., 2020.

[40] M. Pedersen and M. Meneghini, "Quantifying undetected covid-19 cases and effects of containment measures in italy: Predicting phase 2 dynamics," 032020.

[41] K. Z. Ehmann, C. Drosten, C. Wendtner, M. Zange, et al., "Virological assessment of hospitalized cases of coronavirus disease 2019," Nature, vol. 581, pp. 465-469, 2020.

[42] A. L. Bertozzi, E. Franco, G. Mohler, M. B. Short, and D. Sledge, "The challenges of modeling and forecasting the spread of covid-19," Proc. Nat. Acad. Sci., vol. 117, no. 29, pp. 16732-16738, 2020.

[43] S. Romano, A. Fierro, and A. Liccardo, "Beyond the peak: A deterministic compartment model for exploring the covid-19 evolution in italy," PloS One, vol. 15, no. 11, p. e0241951, 2020.

[44] G. Wang, Q. S. Jia, J. Qiao, J. Bi, and M. Zhou, "Deep learning-based model predictive control for continuous stirred-tank reactor system," IEEE Trans. Neural Netw. Learn. Syst., pp. 1-10, 2020.

[45] A. Carron, F. Seccamonte, C. Ruch, E. Frazzoli, and M. N. Zeilinger, "Scalable model predictive control for autonomous mobility-on-demand systems," IEEE Trans. on Control Syst. Tech., vol. 29, no. 2, pp. 635644, 2021.

[46] A. P. Lemos-Paião, C. J. Silva, and D. F. Torres, "A new compartmental epidemiological model for covid-19 with a case study of portugal," Ecol. Complex., p. 100885, 2020.

[47] F. Zhou, T. Yu, R. Du, G. Fan, et al., "Clinical course and risk factors for mortality of adult inpatients with covid-19 in wuhan, china: a retrospective cohort study," Lancet, 2020.

[48] K. Aspelund, M. Droste, J. H. Stock, and C. D. Walker, "Identification and estimation of undetected covid-19 cases using testing data from iceland," NBER Work. Pap., no. w27528, 2020.

[49] "Decision \& Control Laboratory," http://dclab.poliba.it/covid-19, [Accessed 2020-12-29].

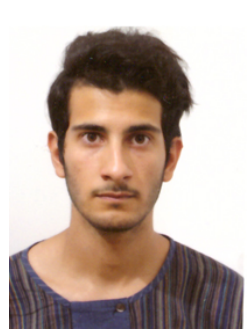

Paolo Scarabaggio (S'20) received the B.Sc. degree in Mechanical Engineering in 2017 and the M.Sc. degree in Management Engineering in 2019 from the Politecnico di Bari, Bari, Italy. In 2019, he was a visiting student at the Delft Center for Systems and Control, TU Delft, The Netherlands.

$\mathrm{He}$ is currently working toward the Ph.D. degree in the Dept. of Electrical and Information Engineering of the same university under the supervision of Prof. Engr. Mariagrazia Dotoli.

His research interests include modeling, optimization, and distributed control of energy and complex networked systems.

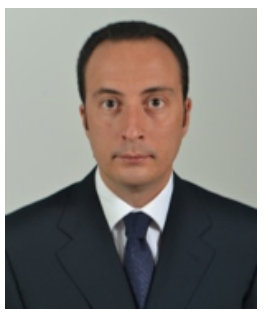

Raffaele Carli (M'17) received the Laurea degree in Electronic Engineering with honours in 2002 and the Ph.D. in Electrical and Information Engineering in 2016, both from Politecnico di Bari, Italy. From 2003 to 2004, he was a Reserve Officer with Italian Navy. From 2004 to 2012, he worked as System and Control Engineer and Technical Manager for a space and defense multinational company.

Dr. Carli is currently an Assistant Professor in Automatic Control at Politecnico di Bari, and his research interests include the formalization, simulation, and implementation of decision and control systems, as well as modeling and optimization of complex systems. He was member of the International Program Committee of 20+ international conferences and guest editor for special issues on international journals. He is author of $60+$ printed international publications.

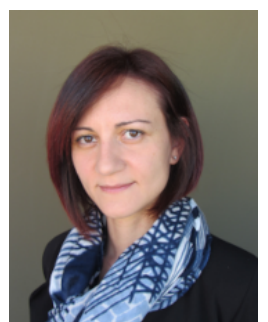

Graziana Cavone (S'16, M'18) received the Laurea degree summa cum laude in Control Engineering in 2013 from the Polytechnic of Bari, Italy and the $\mathrm{Ph} . \mathrm{D}$. degree (excellent with honors) in Electronic and Computer Engineering in 2018 from the University of Cagliari, Italy. She currently is a postdoc Research Fellow at Polytechnic of Bari. She has been Research Fellow in 2014 at Polytechnic of Bari, Italy, and Visiting Ph.D. Student in 2016-2017 at Delft University of Technology, the Netherlands. Her research interests include modelling, simulation, optimization, and control of discrete-event and hybrid systems, distributed control, automated manufacturing systems, intelligent transportation, smart cities. She is the Local Arrangements chair of the 2021 Mediterranean Conference on Control and Automation. She is Associate Editor for the international Journal Results in Control and Optimization (RICO). She was member of the International Program Committee of 20+ international conferences and Guest Editor for special issues on international journals. She was awarded a research grant by the National Science Foundation of China for year 2020.

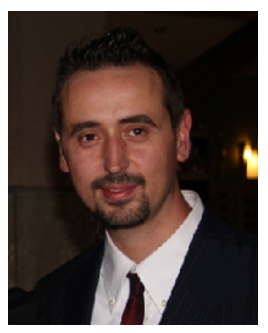

Nicola Epicoco is an Assistant Professor in Automatic Control at the Department of Information Engineering, Computer Science and Mathematics at the University of L'Aquila (Italy). He received the Master's degree in Mechanical Engineering at Polytechnic of Bari and the Ph.D. in Electrical and Information Engineering at the same polytechnic in 2016, focusing on the impact of Information and Communication Technologies on logistics and transportation systems. He is a member of the Center of Excellence on Design methodologies for Embedded controllers, Wireless interconnect and System-on-chip (DEWS) at the University of L'Aquila.

He was Associate Editor or member of the International Program Committee of 20+ international conferences and Guest Editor for special issues on international journals. His research interests include modeling, simulation, optimization, management, and control of dynamic systems, and particularly supply chains, manufacturing systems, traffic networks, transportation systems, energy systems. He is author of $50+$ printed international publications.

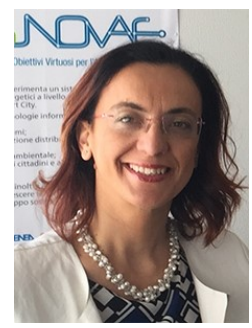

Mariagrazia Dotoli (M'96, SM'12) received the Laurea degree in Electronic Engineering with honours in 1995 and the Ph.D. in electrical engineering in 1999 from Politecnico di Bari (Italy).

She has been a visiting scholar at the Paris 6 University and at the Technical University of Denmark. She is expert evaluator of the European Commission since the 6th Framework Programme. She is a Full Professor in Automatic Control at Politecnico di Bari, which she joined in 1999. She has been the Vice Rector for research of Politecnico di Bari and a member elect of the Academic Senate. Her research interests include modeling, identification, management, control and diagnosis of discrete event systems, manufacturing systems, logistics systems, traffic networks, smart grids and networked systems.

Prof. Dotoli was co-chairman of the Training and Education Committee of ERUDIT, the European Commission network of excellence for fuzzy logic and uncertainty modeling in information technology, and was key node representative of EUNITE, the EUropean Network of excellence on Intelligent Technologies. She is the Editor in Chief of the IEEE SYSTEMS MAN AND CYBERNETICS SOCIETY E-NEWSLETTER, and an Associate Editor of the IEEE TRANS. ON AUTOMATION SCIENCE AND ENGINEERING, of the IEEE TRANS. ON SYSTEMS, MAN, AND CYBERNETICS and of the IEEE TRANS. ON CONTROL SYSTEMS TECHNOLOGY. She is the Program chair of the 2020 IEEE Conference on Automation Science and Engineering and the General chair of the 29th Mediterranean Conference on Control and Automation. She was the Program co-chair of the 2017 IEEE Conference on Automation Science and Engineering, the Workshop and Tutorial chair of the 2015 IEEE Conference on Automation Science and Engineering, the Special Session co-chair of the 2013 IEEE Conference on Emerging Technology and Factory Automation and chair of the National Committee of the 2009 IFAC Workshop on Dependable Control of Discrete Systems. She was member of the International Program Committee of 80+ international conferences. She is author of $200+$ publications, including 1 textbook (in Italian) and 70+ international journal papers. 